\title{
Structure of hydrogen-rich transverse jets in a vitiated turbulent flow
}

\author{
S. Lyra ${ }^{1}$, B. Wilde ${ }^{2}$, H. Kolla ${ }^{1}$, J. Seitzman ${ }^{3}$, T.C. Lieuwen ${ }^{3}$ \& J.H. Chen ${ }^{1}$ \\ ${ }^{1}$ Reacting Flow Research Department, Combustion Research Facility, Sandia National \\ Laboratories, Livermore, CA 94551, USA \\ ${ }^{2}$ School of Mechanical Engineering, Georgia Institute of Technology, Atlanta, GA 30332, \\ USA \\ ${ }^{3}$ School of Aerospace Engineering, Georgia Institute of Technology, Atlanta, GA 30332, \\ USA
}

\begin{abstract}
This paper reports the results of a joint experimental and numerical study of the flow characteristics and flame structure of a hydrogen rich jet injected normal to a turbulent, vitiated crossflow of lean methane combustion products. Simultaneous high-speed stereoscopic PIV and OH PLIF measurements were obtained and analyzed alongside three-dimensional direct numerical simulations of inert and reacting JICF with detailed $\mathrm{H}_{2} / \mathrm{CO}$ chemistry. Both the experiment and the simulation reveal that, contrary to most previous studies of reacting JICF stabilized in low-to-moderate temperature air crossflow, the present conditions lead to a burner-attached flame that initiates uniformly around the burner edge. Significant asymmetry is observed, however, between the reaction zones located on the windward and leeward sides of the jet, due to the substantially different scalar dissipation rates. The windward reaction zone is much thinner in the near field, while also exhibiting significantly higher local and global heat release than the much broader reaction zone found on the leeward side of the jet. The unsteady dynamics of the windward shear layer, which largely control the important jet/crossflow mixing processes in that region, are explored in order to elucidate the important flow stability implications arising in the inert and reacting JICF. The paper concludes with an analysis of the ignition, flame characteristics, and global structure of the burner-attached flame. Chemical explosive mode analysis (CEMA) shows that the entire windward shear layer, and a large region on the leeward side of the jet, are highly explosive prior to ignition
\end{abstract}


and are dominated by non-premixed flame structures after ignition. The predominantly mixing limited nature of the flow after ignition is examined by computing the Takeno flame index, which shows that $\sim 70 \%$ of the heat release occurs in non-premixed regions.

Keywords: DNS, experiment, transverse jet, vitiated crossflow. 


\section{Introduction}

The jet in crossflow (JICF) is a canonical configuration employed in many industrial and transportation combustion systems. It facilitates rapid mixing between two streams, driven primarily by a complex three-dimensional flow field [1]. Considering combustion applications specifically, the JICF is widely encountered in aircraft and electric power generating gas turbine combustors. For lean, premixed systems, JICF technology is used in both the fuel/air mixers/nozzles and the combustor itself. For instance the leanpremixed-prevaporized (LPP) concept of aircraft engine combustor relies on a JICF type fuel injector to quickly mix the vaporizing liquid fuel with gaseous air. Likewise, industrial gas turbine combustors operating with gaseous fuels employ a JICF based fuel premixer. In both of these applications, the JICF provides rapid mixing to enable the primary combustion zone to operate in a lean premixed mode.

The JICF is also used in staged combustors, such as RQL (rich, quickquench lean) systems, in both aircraft and stationary gas turbines where either an air jet mixes with a rich, vitiated crossflow, or a secondary fuel jet issues into lean combustion products from a primary combustion zone. Fuel staging is an attractive design strategy as it can facilitate fuel flexibility and enable load variations while satisfying stringent emissions regulations. However, for fuel premixing the JICF near field flame stabilization is important to flameholding safety (i.e., to ensure that a flame cannot stabilize in the JICF wake if it were to flashback [2]), while for staged combustion, the design concern is primarily that of temperature uniformity into the turbine, $N O_{x}$ emissions from the secondary zone, and JICF thermoacoustics.

The fundamental momentum and scalar transport in non-reacting JICF has been studied extensively both experimentally and numerically. Comprehensive recent reviews are given by Karagozian [3] and Mahesh [4]. Previous work has focused primarily on the influence of momentum flux ratio, density ratio, nozzle geometry, Reynolds number, and boundary layer thickness in inert JICF configurations [1, 5, 6, 7, 8, 9, 10]. Much less is known, however, about the behavior of reacting JICF, particularly at crossflow temperatures relevant in practical devices. Flames can be stabilized by both flame propagation and autoignition processes, depending upon crossflow temperature and fuel composition. Near field flame stabilization in moderate temperature crossflow was studied experimentally [11] and using direct numerical simulations $[12,13]$. Flame blowout limits in JICFs have been 
reported by a number of studies which correlate the lift-off hight with the laminar burning velocity, the jet diameter and the jet and crossflow velocities, $[14,15,16,17]$. Higher temperature crossflows were recently studied experimentally $[18,19,20]$, which emphasized the role of autoignition in flame stabilization at high temperature.

The near field flame structure is also of interest and, in particular, the effect of the spatial heat release distribution on the instantaneous flow field and the shear layer dynamics. As the fuel jet discharges into the crossflow, the shear layer between the windward side of the jet and the crossflow rolls up into concentrated regions of vorticity (the shear layer vortices, SLV) near the exit of the jet injector and amalgamate with downstream distance. The elevated temperature of the vitiated crossflow can make autoignition phenomena very important in the jet near field and lead to a burner-attached flame stabilized in the stagnation region upstream of the windward shear layer. Recent experimental and numerical findings suggest that the character of the windward shear layer, and thus the spatio-temporal dynamics of the SLV, is sensitive to changes in the jet-to-crossflow momentum flux ratio, $J$, and the jet-to-crossflow density ratio, $S$.

Megerian et al. [21] performed hot-wire measurements in the windward shear layer of JICF with different $J$. They observed a dramatic shift in the velocity spectra and a significant concentration of spectral power into a dominant fundamental mode and its harmonics when $J$ was reduced below $J \sim 10$. They interpreted this spectral shift as a transition from convective instability, where the shear layer acts as a noise amplifier, to global instability, where the flow behaves as a self-excited oscillator. Subsequent DNS and global linear stability analysis of non-reacting $J=9$ JICF performed by Bagheri et al. [22] and Schlatter et al. [23] identified two global modes, including a high-frequency mode associated with the windward shear layer and a lower-frequency mode found in the leeward side of the jet. Getsinger et al. [24] extended the earlier hot-wire measurements by Megerian et al. [21] to consider variable density ratio, $S$, transverse jets and found that the JICF exhibited evidence of global instability for $S<0.45$, which is lower than the critical $S$ found for axial jets [25]. The study of Getsinger et al. [24] also reported that the $J$ value at which the convective to global mode transition occurred was independent of density ratio, whereas axial jet studies suggest that the convective-global stability transition is a function of $S$.

In this study, results are presented from a joint experimental-numerical investigation of a hydrogen-rich jet in a vitiated crossflow comprised of prod- 
ucts of methane combustion, using complementary information from experiments and direct numerical simulations (DNS). While the experimental and DNS conditions are not identical, significant effort was put forth to match important fluid mechanic and chemical kinetic parameters, in order to enable mutually useful comparisons of the results.

Despite the fact that JICFs have been widely studied, the configuration has not been examined in its entirety, especially for turbulent vitiated crossflows which lead to nozzle attached flames. To the authors' knowledge this is the first joint experimental and numerical study of vitiated JICFs specifically designed to lie in the same turbulence and thermochemical parameter space aiming to provide complementary insights and to enable understanding of the coupled role of flow, kinetics, and hydrodynamic stability. Since this is the first presentation on this effort particular emphasis has been placed on a thorough description of the experimental facility and diagnostics, the details of the simulation as well as on the analysis of the flame structure and the distinct characteristics of the present complex, reacting JICF. The motivation behind the joint DNS/experimental study is to provide complementary information and establish that the statistical trends describing the flow and flame structure are qualitatively similar. The DNS is then used to glean fine scale information regarding the reacting shear layer dynamics and the mode of combustion in vitiated conditions. Measurements and DNS data from this arrangement can also serve as validation data for a posteriori testing and assessment of LES and RANS combustion models as has been done for low-temperature JICFs [26].

Hence, the main focus of the present work is to investigate the characteristics of a complex reacting jet in crossflow as revealed by high speed laser diagnostics and DNS. The mean flame and flow structure is examined using mutual insights gained from the experimental and DNS data. For example, while jet flames are nominally axisymmetric in the absence of crossflow, there are significant distinctions between windward and leeward flame structures in the JICF which are emphasized - their respective characteristics are reported and compared. Time-resolved DNS data are used to investigate the the unsteady dynamics of the shear layer of the reacting jet in comparison with its non-reacting counterpart. Chemical explosive mode analysis is used to quantify the propensity of the mixture to proceed to thermal runaway in isolation prior to ignition. Finally, the degree of premixedness of the heat release zones are investigated using the Takeno flame index. The remainder of the paper is structured as follows. Section 2 describes the experimental 
facility and the diagnostics used in the experiment. Section 3 describes the DNS code and the physical and numerical parameters of the configuration. The experimental data and the DNS results are presented and compared in Section 4. Finally, the main findings and conclusions drawn from the analysis are summarized in Section 5.

\section{Experimental Facility and Diagnostics}

Two experimental test conditions are considered in the present work, one where the JICF is non-reacting and one where it is reacting. The crossflow is comprised of cold air for the non-reacting condition and is vitiated for the reacting condition. The motivation for this choice will be discussed in more detail in Section 3. The experimental facility utilized for both conditions is comprised of three sections: i) a vitiator, ii) a flow conditioning section, and iii) an optically accessible test section. A schematic of the facility is shown in Figure 1. The vitiator section consists of a natural gas burner coupled to a cylindrical, refractory-lined combustion chamber. In the reacting case, the vitiator is operated at an overall equivalence ratio of $\Phi=0.46$.

Hot product gases from the vitiator flow into the rectangular flow conditioning section and opposed air inlets inject a metered quantity of roomtemperature dilution air to reduce the temperature of the vitiated products to $T=1200 K$. A series of settling chambers and flow straighteners condition the crossflow prior to the test section, where the jet is injected flush with the lower wall. The crossflow enters at $T_{\infty}=300 \mathrm{~K}$ and $T_{\infty}=1200 \mathrm{~K}$ in the non-reacting and the reacting cases, respectively. The Reynolds number of the crossflow in the test section based on mean velocity, $U_{\infty}$, and the hydraulic diameter of channel at the inlet to the test section for the reacting and non-reacting conditions is $R e_{\infty}=10270$ and $R e_{\infty}=40090$, respectively.

The test section dimensions are $127 \mathrm{~mm} \times 76.2 \mathrm{~mm}$ and optical access is provided by quartz windows at the top and sides of the test section. The jet, which has a diameter $d$ of $3.175 \mathrm{~mm}$, is injected normal to the crossflow direction $86 d$ downstream of the entrance to the test section. The jet injector is fabricated from ceramic to minimize quenching and its contour is identical to that used in the study by Megerian et al. [21]. The smooth contraction nozzle generates a thin, nearly tophat velocity profile at the exit.

The jet composition is $70 \% \mathrm{H}_{2} / 30 \% \mathrm{He}$ by volume for the reacting case

and $28 \% \mathrm{~N}_{2} / 72 \% \mathrm{He}$ for the inert case. The jet inlet temperature in the 
non-reacting and reacting cases is $T_{j}=300 \mathrm{~K}$ and $T_{j}=325 \mathrm{~K}$, respectively. The slightly higher inlet temperature in the reacting case is due to heat transfer from the vitiated crossflow through the jet nozzle. The inert and reacting mixture compositions were selected to provide identical jet-tocrossflow density ratios for the inert and reacting cases, $S=\rho_{j} / \rho_{\infty}=0.37$, below the critical value for transition from convective to global instability observed previously in non-reacting JICF studies by Getsinger et al. [24]. The significance of the density ratio and its influence on JICF dynamics will be discussed in the Section 4.2. The jet-to-crossflow momentum flux ratio, $J=\rho_{j} U_{j}{ }^{2} / \rho_{\infty} U_{\infty}^{2}$, is 9 and the jet Reynolds number based on mean jet velocity, $U_{j}$, is $R e_{j}=2420$ in the non-reacting case and $R e_{j}=2475$ in the reacting case.

The jet fluid passes through a choked orifice $40 d$ upstream of the jet injection location to minimize injector coupling between the crossflow acoustics and the jet supply system. Crossflow acoustics during tests are characterized using Kistler 211B5 sensors in a two-microphone method configuration. A third Kistler sensor is located in the jet supply plenum. Finally, the vitiator has been carefully tuned to achieve the lowest possible crossflow acoustics, whose associated flow oscillations are approximately $0.2 \%$ of the crossflow velocity.

Simultaneous high-speed stereoscopic particle image velocimetry (SPIV) and $\mathrm{OH}$ planar laser induced fluorescence (OH-PLIF) were used to characterize the flowfield. Illumination was provided by a dual head, frequency doubled Nd:YLF laser (Litron LDY303-HE) operated at $10 \mathrm{kHz}$, with measured pulse energy of about $2 \mathrm{~mJ}$. The laser beam was expanded, collimated, formed into $\mathrm{a} \sim 1 \mathrm{~mm}$ thick sheet, and guided into the test section using a series of mirrors and converging/diverging lenses (LEO) suspended above the test section. The laser sheet was aligned to the jet centerplane, and the laser pulses for the non-reacting and reacting cases were separated by $21.0 \mu s$ and $8.1 \mu s$, respectively.

SPIV images were captured by two Photron SA5 cameras mounted in a side-scatter configuration. The cameras were positioned approximately $30^{\circ}$ relative to the jet centerplane normal coordinate ( $z$-axis). Each camera was equipped with a $105 \mathrm{~mm}$ focal length lens at $\mathrm{f} / 8$ mounted on Scheimpflug adapters and viewed the test section through a narrow-band interference filter. Standard corrections for perspective and distortion were made using a LaVision 058-5 3D dot target. Camera resolution was $512 \times 512$ and the viewable area was approximately $45 \times 45 \mathrm{~mm}^{2}$. 11000 double-framed image 
pairs were recorded at each test condition $(\sim 1.1 s)$.

Both the crossflow and the jet were seeded with Dupont R-960 $\mathrm{TiO}_{2}$ particles with mean particle diameter of $0.50 \mu \mathrm{m}$. The crossflow seeding system consisted of a passively agitated swirling seeder operated with about $1 \mathrm{~g} / \mathrm{s}$ of air flow, while the jet flow was seeded using a small surface spray type seeder. Seeded crossflow air was injected through the dilution air ports. Seeding density of the crossflow and the jet were manually balanced. Velocity vectors were computed using LaVision DaVis 8.1.6 software with multi-pass processing and adaptive interrogation windows. Initial interrogation windows were $24 \times 24$ with $50 \%$ overlap while final interrogation windows were $12 \times$ 12 with $50 \%$ overlap, resulting in vector spacing of about $0.48 \mathrm{~mm}$.

The high-speed OH-PLIF system consisted of a frequency doubled Nd:YAG laser (Edgewave InnoSlab IS811-E) with output power of $40 \mathrm{~W}$ pumping a tunable dye laser (Sirah Credo LG24). The UV output at $283 \mathrm{~nm}$ had pulse width of about $9 \mathrm{~ns}$ and pulse energy of $0.2 \mathrm{~mJ}$. The dye laser wavelength was tuned to the $Q_{1}(9)$ transition of $O H$ in the $(0,1)$ band of the $A^{2} \Sigma^{+}-X^{2} \Pi$ system. The UV laser beam was expanded, collimated, and combined with the SPIV sheet. Both laser sheets were approximately $50 \mathrm{~mm}$ wide. The OH-PLIF laser sheet was focused to a thickness of about $0.4 \mathrm{~mm}$.

The fluorescence signal near $310 \mathrm{~nm}$ was collected using a Photron SA1.1 camera optically coupled to a Lambert Instruments HiCatt 25 intensifier and a $45 \mathrm{~mm}$ Cerco $f / 1.8$ lens. The OH-PLIF camera was located in between the SPIV cameras and normal to the jet centerplane. The intensifier was gated to $200 \mathrm{~ns}$ and the timing of the cameras and lasers for both systems were controlled using a BNC Model 575 timing unit. Background flame luminescence was blocked using a narrow-band interference filter. 9700 images were recorded per OH-PLIF data set and the resolution was $768 \times 768$. The viewable area was similar to that of the SPIV and standard corrections for perspective and distortion were applied using images of the 3D target. Laser sheet intensity variations along the x-direction were corrected using an acetone calibration procedure, and the final OH-PLIF images were corrected for background $O H$, which was generally very low ( $<10$ counts).

\section{Mathematical Description and Configuration}

The DNS are performed using the code S3D [27] developed at the Combustion Research Facility at Sandia National Laboratories. In addition to 
the previous JICF studies [28, 29], S3D has been used for a range of studies, including premixed flames $[30,31,32]$, non-premixed flames [33, 34] and auto-ignition [35, 36]. S3D solves the compressible formulation of continuity, Navier-Stokes, total energy and species conservation equations on a structured 3D Cartesian grid using an explicit eighth-order centered finite difference scheme for evaluation of spatial derivatives and a fourth-order, sixstage explicit Runge-Kutta scheme for time advancement. S3D implements finite rate chemical kinetics and mixture averaged transport coefficients by interfacing with CHEMKIN and TRANSPORT libraries [37,38]. The chemical reactions in the gas phase are described by a detailed mechanism for $\mathrm{H}_{2} / \mathrm{CO}$ combustion [39], involving 13 species and 35 reactions. Nitrogen is assumed to be inert and $N O_{x}$ formation reactions are not considered. The extent of the computational domain is $45 \mathrm{~mm} \times 44.5 \mathrm{~mm} \times 44.5 \mathrm{~mm}$ in the streamwise $(x)$, transverse $(y)$ and spanwise directions $(z)$, respectively. Navier-Stokes characteristic boundary conditions (NSCBC) are imposed and the streamwise boundaries are treated as non-reflecting inflow and outflow, while the spanwise boundaries are specified as periodic. The transverse boundaries are treated as adiabatic, no-slip, inert walls. A transverse jet of diameter, $d=1.5 \mathrm{~mm}$, issues vertically from the lower boundary and its centre is located at $5.5 \mathrm{~mm}$ from the upstream $x$ boundary at the spanwise midplane. The mean jet velocity is represented by symmetric smoothed top-hat profile, with a momentum thickness of $\theta / d=0.073$. This is consistent with earlier DNS simulations of JICFs $[12,13]$ and similar to the profiles in the experiments by Megerian et al. [21] where an identical nozzle has been used. The crossflow corresponds to a fully developed turbulent channel flow comprised of combustion products of lean methane.

Figure 2 presents the DNS configuration from an instantaneous volume rendering of $\mathrm{H}_{2} \mathrm{O}$ and $\mathrm{H}_{2}$ mass fractions and temperature. The DNS conditions were set up to match the experimental conditions as closely as possible. The jet and crossflow compositions and the physical and numerical parameters have been selected so that the momentum flux ratio, the density ratio, and the Damköhler number are as close as possible to the reacting jet experiment. To ensure that the Damköhler number, defined based on the jet and ignition time scales of the stoichiometric mixture, matches that of the experiment, the jet and crossflow temperatures have been adjusted to $407.26 \mathrm{~K}$ and $1640 \mathrm{~K}$, respectively. The jet and crossflow Reynolds numbers are matched as closely as possible between the experiments and simulations. Practical limitations dictate that the crossflow Reynolds numbers vary in 
the non-reacting condition. In particular, in the simulation the chemistry is "turned-off", thereby enabling the investigation of a non-reacting $\mathrm{H}_{2} / \mathrm{He}$ jet in a vitiated crossflow. Since it is not possible to "turn-off" chemistry in the experiment, a non-reacting jet condition was achieved by changing the composition of the jet ( $72 \% \mathrm{He} / 28 \% \mathrm{~N}_{2}$ by mole) and reducing the crossflow temperature. Thus, although all of the other important JICF parameters are matched, an unavoidable consequence of this decision is that the crossflow Reynolds number is higher in the non-reacting experimental condition than in the simulation. For the reacting cases the Reynolds numbers are matched to within 5\%. The physical test conditions for the experiment and the DNS are summarized in Table 1.

As alluded to earlier, the elevated crossflow temperature leads to small ignition delay times, i.e. $t_{i g n} \sim 10^{-6} \mathrm{~s}$ for a stoichiometric mixture. This, in turn, leads to initiation of reactions very close to the jet exit as the fuel issues from the nozzle tip and mixes with the crossflow, so that the flame is essentially attached to the jet outlet. The significant concentration of $\mathrm{H}_{2} \mathrm{O}$ surrounding the jet in the near field denotes the development of a stable flame that encompasses the fuel plume and spans both the windward and leeward sides of the jet.

The time-dependent fluctuating velocity field imposed at the inflow boundary is obtained from an auxiliary inert fully developed turbulent channel flow simulation. The auxiliary channel flow simulation is performed on a domain that is $178.3 \mathrm{~mm} \times 44.5 \mathrm{~mm} \times 44.5 \mathrm{~mm}$ in the $x, y$ and $z$ directions, respectively and with a grid of $1000 \times 720 \times 240$ points, respectively. The grid spacing is uniform in the $x$ and $z$ directions while it is refined in the $y$ direction to ensure that the near-wall boundary layer is sufficiently resolved, with the first point located at $y^{+} \sim 0.25$, and 30 points below $y^{+} \sim 10$. The auxiliary channel flow simulation is run for five flow through times, based on the centerline velocity, before it is fully developed. It is subsequently advanced for five more flow through times to ensure a sufficient number of samples are available to provide the time-dependent inlet velocities to the inert and reacting simulations. The inert JICF simulation is initially performed for one flow through time to allow for the hydrogen plume to be established. Subsequently the inert JICF solution is advanced for three more flow through times. In parallel, a reacting JICF DNS is performed in which the plume is ignited and the simulation is advanced at an intermediate resolution until the ignition transient is flushed out of the computational domain. The solution is then up-sampled to the production grid with $1500 \times 2080 \times 1500$ points in 
the $x, y$ and $z$ directions, respectively. The lateral boundaries in the JICF simulations are located by design to be sufficiently far from the jet so that they do not interact with the plume or flame and are specified as periodic for consistency with the inflow channel flow solution $[12,13]$. Both the inert and reacting JICF simulations are performed on computational domains that are refined in the wall-normal $(y)$ direction to ensure that the near-wall boundary layer is sufficiently resolved. The grid spacing is uniform in the $x$ and $z$ directions in the jet near field whereas a mild stretching is employed in the far field. In detail, for the inert JICF simulation the first point off the wall is at $y^{+}=0.5$ where the superscript + indicates normalization by the viscous length scale, $\delta_{\nu} \sim 89 \mu \mathrm{m}$. There are two points within $y^{+}=1$ and 15 points within $y^{+}=10$ to satisfy the resolution requirements in the viscous layer $[40,32]$. A refined grid has been used in the near field $(x / d<10)$ in the streamwise direction and for $10 d$ around the jet in the spanwise direction where the grid spacing is uniform and equal to $x^{+}=z^{+}=0.77$. The same refinement strategy has been used in all three directions of the reacting JICF simulation with the grid resolution carefully chosen to adequately resolve both the flame and the turbulence fields. The near wall resolution was obtained by the viscous length scale whereas the required flame resolution was determined from a one-dimensional flame grid convergence study and ensured that all radical profiles and reaction rates were sufficiently resolved with a minimum of 15 grid points across the thinnest species mass fraction layer. Therefore the first point off the wall was at $y^{+}=0.2$, and 50 points are placed within $y^{+}=10$. A refined grid has been used in the near field $(x / d<10)$ in the streamwise direction and for $10 d$ around the jet in the spanwise direction where the grid spacing $x^{+}=z^{+}=0.2$. The calculations are performed on Titan at ORNL and Edison at NERSC on more than $10^{5}$ cores.

Table 1: Physical parameters of the JICF experiments and DNS

\begin{tabular}{|c|c|c|c|c|c|c|c|c|c|c|c|}
\hline Case & R/NR & Jet Composition & $\mathbf{J}$ & $\mathrm{S}$ & $\mathbf{T}_{\mathbf{j}}[\mathbf{K}]$ & $\mathbf{T}_{\infty}[\mathbf{K}]$ & $\mathbf{R e}_{\mathbf{j}}$ & $\operatorname{Re}_{\infty}$ & Da & $\mathrm{U}_{\mathbf{j}}[\mathrm{m} / \mathrm{s}]$ & $\mathrm{U}_{\infty}[\mathbf{m} / \mathbf{s}]$ \\
\hline $\operatorname{Exp}$ & NR & $28 \% / 72 \% \quad N_{2} / H e$ & 9 & 0.37 & 300 & 300 & 2420 & 40090 & {$[-]$} & 35.1 & 7.1 \\
\hline $\operatorname{Exp}$ & $\mathrm{R}$ & $70 \% / 30 \%$ & 9 & 0.37 & 325 & 1200 & 2475 & 10270 & 1 & 94.1 & 19.1 \\
\hline DNS & NR & $70 \% / 30 \%$ & 9 & 0.37 & 407.26 & 1640 & 2420 & 9480 & [-] & 291 & 59 \\
\hline DNS & $\mathrm{R}$ & $70 \% / 30 \% \quad \mathrm{H}_{2} / \mathrm{He}$ & 9 & 0.37 & 407.26 & 1640 & 2420 & 9480 & 1 & 291 & 59 \\
\hline
\end{tabular}




\section{Results and Discussion}

The results presented in this paper are organized around three main issues. First, we discuss the time averaged flow and flame structures. This section focuses on the very distinct flame characteristics on the windward and leeward sides of the jet, due to the significant differences in flow strain. Then, we utilize the DNS results to extract vorticity spectra from selected locations in the nearfield windward shear layer to probe the stability of the reacting jet and to determine the mode of combustion, as well as quantify the Takeno index to show the dominance of non-premixed flame structures in driving the majority of the JICF heat release.

\subsection{Time-averaged Flow and Flame Structure}

Reynolds-averaged flame and flow features on the spanwise midplane of the non-reacting JICF are presented in Fig. 3, enabling comparison of the averaged velocity magnitude between the experiment and the DNS. Note that the velocity is not resolved below $y / d=0.75$ in the experiment due to laser reflections off the lower wall. Recall that, though the $J$ values are matched, the crossflow Reynolds numbers of the experiment and DNS are different for the non-reacting case (having values of 40200 and 9480, respectively), and so comparisons should only be qualitative. As the experimental crossflow Reynolds number is higher by a factor of four, its shorter potential core $(y / d<2)$ relative to the DNS $(y / d=3)$ is expected. In addition, the trajectory of the experimental result turns more quickly into the crossflow than the DNS. As JICF trajectories are largely independent of Reynolds number for $R e \geq 1 \times 10^{3}$ [8], this difference in trajectory may be a manifestation of a thinner approach flow boundary in the experiment further discussed below.

Figure 4 compares the Reynolds averaged velocity magnitude and $\mathrm{OH}$ mass fraction fields on the spanwise midplane of the reacting JICF. The mean heat release rate isolines, spanning $10 \%$ to $90 \%$ of the maximum value, are superimposed on the DNS results to demarcate the reaction zone. The two-dimensional contour plots show that the major flow and flame features including the jet trajectory and spatial distribution of $O H$ extent are qualitatively similar between the reacting DNS and experiment (they are more directly compared in Fig. 5). In particular, the presence of significant heat release rate on the jet windward side shows that a thin reaction zone is formed in the mixing layer between the crossflow and the jet near field, $y / d<3$, that leads to an attached flame established near the jet exit. The peak volumetric 
heat release rate occurs at approximately $y / d \sim 0.75$, within a region where the average mixture fraction is nearly stoichiometric $\bar{\xi}=0.04\left(\bar{\xi}_{s t}=0.03\right)$, at the outer edge of the jet shear layer (the vorticity magnitude is $\sim 2 \%$ of the maximum value) where the average velocity is relatively low, $\bar{U} / U_{j} \sim 10 \%$. A much broader region of elevated $O H$ is observed in the near field of the leeward side of the jet. Note that the two flame branches remain firmly attached to the burner outlet at all times, and detachment from the wall was not observed in either the experiment or the simulation. The low velocity region formed in the jet lee side at $x / d \sim 4$ and $y / d \sim 2$ in the inert JICF persists for reacting conditions. The mean volumetric heat release rate in the leeward side is lower than the mean volumetric heat release rate observed on the windward side by a factor $\sim 2.5$. This is due to the lower gradients, strain rate and scalar dissipation rate of the non-premixed flame on the leeward side as will be presented below. The windward-biased heat release distribution is noteworthy since entrainment of crossflow fluid into the jet plume was shown previously to occur predominately on the leeward side in non-reacting JICF [9], at least for the higher momentum ratio $(J=32.49)$ conditions investigated in that study. More detailed analysis of the heat release distribution in the present work will be presented below.

It is noteworthy that the mean flame characteristics observed here are considerably different than those of the non-vitiated crossflow case of Grout et al. [12], which was studied using the same DNS code and methodology. In that study, the flame was anchored on the jet lee side in a low velocity region coincident with near stoichiometric mixture conditions. No distinct windward flame branch was observed, although an intermittent lifted windward branch, presumably due to propagation of the flame from the lee side stabilization point around the jet, was reported from experiments in [11], similar to the higher-temperature measurements reported by Sullivan et al. [20]. Furthermore, intense turbulent mixing in the near field resulted in partially premixed flame stabilization near the anchoring location. Slight changes in flow conditions, such as through modification of the injection angle, disrupted the flame anchoring and resulted in blowoff [13]. In contrast, under the current vitiated conditions, the flame is uniformly stabilized adjacent to the jet exit around its entire circumference.

Figure 5 compares the time-averaged jet trajectory (center streamline) and the mean streamwise and transverse velocity components along this trajectory from the reacting experiment and DNS. The jet trajectory can be used to define the local arc length coordinate, $s_{j}$, and the local normal co- 
ordinate, $n . \quad n$ is zero at the center streamline, positive in the leeward and negative in the windward sides of the jet, respectively. In the near field, for $x / d \leq 5$, the computed jet trajectory and the transverse velocity component exhibit good agreement with the measurements. The streamwise velocity component, however, increases more rapidly in the DNS than in the experiment, presumably due to differences in the stagnation pressure along the windward edge of the jet between the simulation and the experiment.

Figure 6 compares the measured and computed mean streamwise velocity profile as a function of the distance from the wall, $y / d$, at a streamwise distance, $x / d=1.33$, upstream of the jet center, for reacting conditions. Results from measurements and DNS conducted with and without the transverse jet are presented. In the DNS the mean streamwise velocity is nearly parabolic, corresponding to a fully developed channel flow and is not significantly altered by the presence of the jet. The measured streamwise velocity profile in the absence of the transverse jet is similar although it is approximately $10 \%$ higher than the DNS profile for $y / d>2.5$. The measured crossflow velocity profile in the presence of the jet, however, is lower by up to $30 \%$ at $y / d \sim 5$, at all $y / d$ locations. Therefore the transverse jet in the experiment interacts with a crossflow characterized by a streamwise velocity profile that is lower than in the DNS; thus, both the measured near field streamwise velocity and far field jet turning are lower in the the reacting experiment compared to the DNS.

Figure 7 presents the Reynolds-averaged streamwise velocity, transverse velocity and $\mathrm{OH}$ mass fraction profiles, as a function of the normal coordinate, $n$, at selected locations along the jet trajectory, $s_{j}$, shown in Fig. 5 . The overall agreement of the computed Reynolds-averaged transverse velocity profiles with the experimental data in the jet near field, $s_{j} / d<5$, is good, suggesting that the imposed jet boundary conditions are adequate. Consistent with the results presented in Fig. 5, the streamwise velocity $U / U_{j}$ in the near field, however, is higher in the DNS than in the experiment, and thus the simulated jet is found to exhibit a faster alignment with the crossflow. In the far field, for $s_{j} / d>5$, the streamwise velocity agrees with the measurements but the transverse velocity is slightly underestimated. The $O H$ mass fraction profiles are consistent with the earlier plots showing that the attached flame is characterized by a thin $O H$ layer thickness on the windward side of the jet near the jet exit which becomes broader in the far field. On the lee side of the jet, the width of the reaction zone is broader but does not change significantly along the jet trajectory. It should be noted that the normalized 
$\mathrm{OH}$ PLIF signal from the experiment and the normalized $O H$ mass fraction from the DNS are distinctly different quantities and they are not expected to agree quantitatively. In fact the differences in the crossflow temperature affect the $O H$ formation as it is manifested primarily on the leeward side of the jet. However, they can be used to provide analogous information about the flame topology and the comparison of the experimental and numerical results shows that under the investigated conditions the statistical trends describing the flame structure are qualitatively similar. Specifically, both the experiment and DNS show the significant difference in scale of the $O H$ layer thickness on the leeward and windward sides near the jet exit.

The $O H$ layer thickness and heat release, $q$, layer thickness are presented in Fig. 8 as a function of the arc length trajectory on both sides of the jet. The $O H$ and heat release layer thickness is evaluated as the full width at half maximum of the $O H$ mass fraction and $q$ profiles. Consistent with the $O H$ profiles shown in Fig. 7, the $O H$ layer thickness on the lee side of the jet is an order of magnitude higher than the windward side at the jet exit. As the jet aligns with the crossflow, however, the difference between the thickness of the two flame branches progressively diminishes. Note the close agreement between the DNS and experiment for the evolution of the windward thickness with downstream distance. On the leeward side, the two differ substantively near the jet exit but then attain better agreement further downstream. In the near field $s_{j} / d<5$, the experimentally measured $O H$ layer thickness follows the heat release layer thickness extracted from the simulation along the leeward side of the jet but the two thicknesses diverge in the far field.

The normalized integral of the burning rate $(Q)$ and fuel consumption rate $\left(R R_{H_{2}}\right)$ at selected locations normal to the jet trajectory evaluated on the jet windward and leeward sides are presented in Fig. 8. The integrated burning and fuel consumption rates normalized by their respective global maximum values $\left(q_{\max }\right.$ and $\left.r r_{H_{2} \max }\right)$ and the local $O H$ layer thickness, $\delta_{O H}\left(s_{j}\right)$, are evaluated as:

$$
Q=\frac{\int_{0}^{\infty} q(n) d n}{q_{\max } \delta_{O H}\left(s_{j}\right)}
$$

and

$$
R R_{H_{2}}=\frac{\int_{0}^{\infty} r r_{H_{2}}(n) d n}{r r_{H_{2} \max } \delta_{O H}\left(s_{j}\right)}
$$

and are found to be high in the near field of the windward side and decrease in the far field. The heat release and consumption rate from the leeward side 
is substantially lower than the windward side up to $s_{j} / d \sim 8$. Note also that the leeward side heat release rate exhibits significantly less variation with downstream distance than the windward side.

Further insights into the different characteristics of the leeward and windward sides can be gained from the conditional mean heat release rate and mixture fraction scalar dissipation rate. These quantities are presented in Fig. 9 in the near field $(y / d<3)$ for the windward and leeward sides of the jet. Also shown are one-dimensional laminar flame results, obtained using OPPDIF [41], corresponding to low $(6001 / s)$ and high strain rates $(90001 / s)$. These two levels of strain rate were selected as they closely match the maximum conditional heat release on each side. The mixture fraction conditioned heat release rate is higher on the windward side and exhibits large conditional fluctuations, which suggests that the flame in the vicinity of the windward near field is subject to a broad range of scalar dissipation rate levels. The conditional mean and rms mixture fraction scalar dissipation rate on the windward and leeward jet sides are presented in Fig. 9 (bottom). The conditionally averaged scalar dissipation rate is of interest because of its use in various modeling approaches. The stoichiometric mixture fraction dissipation rate and its variance on the windward side is two-orders of magnitude higher than on the leeward side. For $\xi>0.2$, however, the mean scalar dissipation rate is comparable on both sides, albeit slightly higher on the lee side. The rms of the conditional scalar dissipation is higher on the windward side, accounting for higher heat release rate levels compared to the lee side, and consistent with the one-dimensional strained laminar flame results.

The heat release rate conditioned on the dissipation rate located at the stoichiometric mixture fraction and the pdf of the stoichiometric mixture fraction dissipation rate on the windward (W) and leeward (L) sides of the jet, at $y / d<3(\mathrm{~W} 1, \mathrm{~L} 1)$ and $3<y / d<6$ (W2, L2) are presented in Fig. 10 and Fig. 11, respectively. Values of elevated heat release rate correspond to conditions on the windward side of the jet characterized by strong gradients and high mixture fraction dissipation rate. The corresponding pdfs of the stoichiometric mixture fraction dissipation rate describing the magnitude of the fluctuations about the conditional mean are presented in Fig. 11. The pdf of the conditional scalar dissipation rate is often assumed to follow a lognormal distribution [42], which appears to be a reasonable approximation at most of the locations. The variance of the dissipation rate on the near field windward side is up to three times higher than on the leeward side of the jet. As the sampling window is moved from $y / d<3$ (1) to $3<y / d<6$ 
(2) the mean scalar dissipation rate increases by $50 \%$ on the windward side and is less broadly distributed since its variance reduces by $54 \%$. In the far field of the jet leeward side both the mean scalar dissipation rate and its variance decrease by nearly an order of magnitude compared to the near field averages.

\subsection{Flow Stability Implications}

This section considers the unsteady features of the flow, as well as its hydrodynamic stability. Due to the stagnation flow on the windward side of the jet, the velocity and scalar gradients have their highest average values here. The vorticity in the shear layer between the windward side of the jet and the crossflow quickly concentrates into shear layer vortices (SLV) near the exit of the jet injector. The combination of the stagnation flow and this rollup stretches the material interface between the jet and crossflow, driving up scalar gradients and leading to the larger heat release rates on the windward side of the jet. The shear layer on the leeward side of the jet also rolls up, but much farther downstream, presumably because the shallower velocity gradients lead to slower growth rate of disturbances. The Kelvin-Helmholtz instability is often attributed as the physical mechanism responsible for the formation of the SLV, which can be seen clearly in the instantaneous contour plots of spanwise vorticity shown in Fig. 12.

The degree to which heat release influences hydrodynamic stability and the shear layer growth rate of the reacting JICF is dependent on the location of the flame relative to the shear layer. Clemens \& Paul [43] examined shear layer growth rates in inert and reacting co-axial jets and demonstrated that the reduced growth rates seen in reacting jets were very similar to the reduced growth rates measured in inert jets injected into lower density coflow. Erickson et al. [44] and Emerson et al. [45] studied the effect of reactant preheat temperature in bluff-body stabilized premixed flames and found that increasing preheat temperatures had a strongly destabilizing effect on the reacting wake, which they attributed to a reduction in the density of the reactants relative to the burnt products in the wake. Tacina \& Dahm [46] developed a general equivalence principle to extend scaling laws derived for non-reacting turbulent shear flows to reacting flows. Their results support the notion that combustion heat release modifies shear layer growth rates in momentum-driven flows primarily through the same physical mechanism at work in density stratified inert flows. 
In density stratified, single shear layers with a fixed velocity ratio, it is known that high velocity regions containing higher/lower density fluid lead to lower/higher shear layer growth rates [47, 48]. The interaction between multiple shear layers, however, as is the case in jets and wakes, can lead to fundamentally different stability characteristics. For example, jet and wake flows can contain pockets of absolute instability, which may lead to global instability of the flow [49]. In jet/wake flows, it is known that a low density central region increases/decreases absolute instability growth rates, as shown by Monkewitz \& Sohn [25], Sreenivasan et al. [50], and Yu \& Monkewitz [51]. Jets comprised of low molecular weight fluid generally have different growth rates than heated jets with the same density ratio, which was explained by Raynal et al. [52] as a consequence of differences in the relative offset of the velocity and density gradients in each case. Similarly, in a recent study on the wake characteristics of a turbulent, bluff-body stabilized premixed flame, Emerson et al. [45] showed that, in addition to the magnitude of the density change, the relative locations of velocity and density gradients strongly influence the hydrodynamic stability of the wake flow. In particular, they showed that offsets between these gradients increased absolute instability growth rates, largely by modifying baroclinic vorticity production processes. It is important to note that, because they studied the stability characteristics of a low density wake, increased offset between the inflection point of the velocity and density profiles had a destabilizing effect, as opposed to the stabilizing effect observed previously in jets.

Finally, an unexplored topic is the role of flame stabilization, and therefore kinetic coupling in determining hydrodynamic stability tendencies of shear flows. For example, reacting jets in low-to-moderate temperature air crossflows stabilize a flame on the leeward side of the jet in a well-mixed, low velocity region removed from the lower wall $[5,12]$. The windward shear layer near the jet-exit region may be essentially isothermal under these conditions. Jets injected into high-temperature, vitiated crossflows, on the other hand, lead to strongly attached flames anchored at the jet injector exit in the windward and leeward shear layers. The flame and its location relative to the jet shear layers in the present work can be seen in Fig. 12, which presents instantaneous vorticity fields from the experiment and the simulation superimposed with isolines of $\mathrm{OH}$ mass fraction (DNS)/normalized $\mathrm{OH}$ PLIF (Exp.) to denote the flame location. While combustion heat release near the jet injector would generally be expected to significantly modify the density gradient in the shear layer, this effect is minimal in the present work 
due to the high temperature of the vitiated crossflow and the low stoichiometric mixture fraction. To appreciate the similarity between the density profiles of the inert and reacting cases, Fig. 13 presents mean density gradient profiles extracted along trajectory normal lines at $s_{j} / d$ of 0.5 and 2.0 . The mean vorticity profile has been superimposed to illustrate the location of the peak time-averaged vorticity relative to the positions of the density inflection points. Counter-intuitively, the results show strong similarities between the non-reacting and reacting DNS in the near field. To understand these similarities, it is important to consider the high temperature of the vitiated crossflow and the low stoichiometric mixture fraction $(\sim 0.03)$ for the present conditions. For these conditions, the fluid in the reaction zone is actually more dense than the pure jet fluid, and the attached flame has only a slight effect on the density gradient. Thus, the density gradient in both the inert and the reacting case are primarily controlled by jet/crossflow mixing and heat transfer from the higher temperature crossflow to the lower temperature jet. These effects lead to a pair of inflection points in the windward and leeward density profiles, as shown in Fig. 13. The inflection points located on the crossflow side of the peak vorticity are due to jet/crossflow mixing, while the inflection points closer to the jet centerline are caused by heat transfer to the colder jet fluid from the high temperature crossflow. The density gradient is stronger on the windward side of the jet, and assuming that the axial jet stability results discussed above can be applied here, the larger density jump in the windward shear layer may contribute to its faster roll-up relative to the leeward shear layer, as seen in Fig. 12. Future work will explore the differences between the windward and leeward shear layer stability in more detail.

The present work builds on the previous studies described above to explore the influence of near-field heat release on the stability characteristics of the windward shear layer. Note that the present test conditions lie in the globally unstable parameter space for a non-reacting JICF suggested by Megerian et al. [21] and Getsinger et al. [24]. These studies report that in the simulated flow regime the global mode oscillates at a Strouhal number, defined as $S t=f d / U_{j}$, of $S t \sim 0.7$. This frequency is much higher than can be resolved experimentally $\left(f_{\text {Nyquist }}=5 \mathrm{kHz}\right)$, but can be analyzed from the computations. For this analysis the spanwise vorticity in the windward shear layer of the inert and the reacting JICF are sampled at three distinct locations. The inert case is sampled at $10 \mathrm{~ns}$ intervals for $0.8 \mathrm{~ms}$, while the reacting case is sampled at $4 \mathrm{~ns}$ intervals for $0.8 \mathrm{~ms}$. The sampling inter- 
vals include more than 100 periods of the higher-frequency phenomena (i.e., the shear layer vortices) to insure that the shear layer modes are adequately resolved. The first probe is placed at $(x / d=2.9, y / d=1)$, the second is placed at $(x / d=3.1, y / d=2)$, and the third at $(x / d=3.4, y / d=3)$. The frequency spectra from the inert case are presented in Fig. 14. The spectrum at the first probe location is characterized by a dominant peak corresponding to a Strouhal number $S t \sim 0.35$ and a weaker narrow-band feature at $S t \sim 0.70$. Higher harmonics separated by $S t \sim 0.35$ intervals are apparent further downstream at probe locations 2 and 3 . The peak at $S t \sim 0.35$ remains dominant at all three probe locations, but the relative amplitude of the higher harmonics varies with probe location. To investigate the effect of combustion heat release on the shear layer stability, vorticity spectra from the reacting simulation are presented in Fig. 15. Interestingly, the dominant spectral peak in the reacting case occurs at the same Strouhal number $S t \sim 0.35$ identified in the inert case. On the other hand, the higher frequency peak at $S t \sim 0.70$ is noticeably stronger in the reacting case, and the sequence of regularly spaced harmonics seen in the inert case is obscured by significant background noise. The noisier spectra in the reacting case are likely related to unsteady modification of the shear layer density gradient and vorticity by inherently unsteady combustion processes which disperse energy over a broader range of frequencies compared to the inert case. Relatively small changes to the strength of the density gradient as well as the position of the density gradient relative to the shear layer as a consequence of combustion can act as a parametric noise source, which, in turn, tends to disrupt the periodicity of the shear layer relative to the inert case. It is important to note, however, that the higher frequency mode at $S t \sim 0.70$ observed in both the inert and the reacting spectra corresponds well to the fundamental shear layer instability frequency observed by Megerian et. al [21] and Getsinger et al. [24], which suggests that the lower frequency mode at $S t \sim 0.35$ may be a subharmonic. This would indicate that the fundamental mode saturates very near the jet injector $\left(s_{j} / d<1\right)$ followed by a very rapid transfer of energy to the subharmonic mode. Such rapid transfer of energy to the subharmonic is not generally expected in globally unstable flows but similar behavior was found previously in the non-reacting JICF experiments by Getsinger et al. [24]. Under conditions believed to be globally unstable, those authors observed enhanced energy transfer to the subharmonic mode in low density JICF that was not seen in iso-density JICF. 


\subsection{Combustion Mode}

As has been discussed above, the conditions considered in the present JICF configuration lead to small ignition delay times, i.e. of the order of microseconds, which in turn, promote reactions very close to the jet exit as the fuel issues from the nozzle tip and mixes with the crossflow. Hence, the flame is essentially attached to the jet outlet. Chemical explosive mode analysis (CEMA) [53] can serve as a useful diagnostic to detect the regions where the thermochemical state favours flame anchoring i.e. in the nonreacting DNS and demarcate the locus of the diffusion flame i.e. in the reacting DNS. The CEMA analysis evaluates a chemical explosive mode $\left(t_{\text {exp }}\right)$ as the eigenmode associated with the largest eigenvalue of the Jacobian of the chemical reaction rates, with a positive real part. The explosive mode, $t_{\text {exp }}$, is a chemical property of the mixture, has units of inverse time, and represents the rate-controlling chemical time scale for reaction. CEMA is consistent with conventional practice where a chemical time scale is determined from a reaction rate contributing to a selected species, without the ambiguity associated with the fact that a single species is assumed representative of all chemical time scales $[53,12]$. A positive value of $t_{\text {exp }}$ indicates that the mixture is "explosive" and will effectively transition to burning under adiabatic conditions and in the absence of transport while negative $t_{\exp }$ values delineate non-explosive (burnt) mixtures that would not proceed to thermal runaway in isolation. Since the analysis does not take into account transport, positive explosive modes demarcate flow regions where the mixing between fuel and oxidizer and thermochemical state is sufficient to support combustion. This property of $t_{\text {exp }}$ is utilized here to unambiguously detect and demarcate unburnt and burnt regions and to provide chemical insight and distinguish between the explosive conditions in the non-reacting DNS and the non-explosive diffusion flame once reactions are activated. In detail, the distribution of the chemical explosive mode $\left(t_{\text {exp }}\right)$ is shown in Fig. 16 at the spanwise midplane from the inert and reacting DNSs at a given instant. In the inert case fast chemical modes are present in the jet near field, encompassing both the windward and the lee sides. Locally, the crossflow is comprised of burnt products in equilibrium, and thus exhibits negative explosive modes since it is non ignitable. In contrast, in the reacting case a flame envelope immediately forms around the jet anchored at the nozzle tip, and the flame front is characterized by a negative explosive mode $\left(t_{\text {exp }}\right)$ indicating an almost instantaneous transition to burnt conditions. The dark blue zones $\left(t_{\exp }<<0\right)$ delineate the strong diffusion flame branches in which 
reactions take place, but where the mixture would not proceed to thermal runaway in isolation. To further examine the nature of the flame, a local Damköhler number, defined as $D a=\operatorname{sign}\left(t_{\text {exp }}\right) \times \log _{10}\left[\max \left(1,\left|t_{\text {exp }} \cdot \omega^{-1}\right|\right)\right]$, where $|\omega|$ is the vorticity magnitude, is shown on the spanwise midplane in Fig. 17. Values of $D a<<1$ are associated with the slowest decaying mode in a nonexplosive mixture. The local Da within the region surrounding the jet is much larger than unity indicating the flame is mixing-limited.

The Takeno index [54] defined as $F I=\frac{\nabla Y_{F} \cdot \nabla Y_{O}}{\left|\nabla Y_{F}\right| \cdot\left|\nabla Y_{O}\right|}$, where subscripts $F$ and $O$ denote the fuel and oxidizer, is a useful tool for analyzing the nature of mixing in the flame; the flame index is +1 when the reactants are aligned and -1 when they are opposed, respectively. The flame index and the isolines of heat release shown in Fig. 17 illustrate that the fuel and oxidizer gradients are misaligned in regions where chemical reactions prevail, therefore identifying diffusion as the predominant combustion mode. Distinct from flames stabilized in moderate temperature crossflow where partially premixed combustion is significant, in the present JICF combustion occurs almost entirely in the diffusion mode and this finding is consistent in the near and far field both on the windward and leeward sides of the jet. This is a distinct aspect of the present DNS compared to earlier computational studies performed at low-temperature crossflows $[12,13]$ in which a single flame branch is stabilized on the jet leeward side and where the mode of combustion is partially premixed. Moreover, experiments [11] report the presence of an intermittent lifted windward branch. The conditional heat release rate conditioned on the Takeno index and the pdf of the Takeno flame index on both sides of the jet, in the near and far fields are shown in Fig. 18. The pdf of the Takeno flame index shows that that $70 \%$ of the combustion occurs under conditions in which the alignment between fuel and oxidizer gradients exceeds $115^{\circ}$

\section{Conclusions}

The structure of inert and reacting hydrogen rich transverse jets injected into a turbulent, vitiated crossflow of lean methane combustion products is investigated experimentally using high speed laser diagnostics and numerically using DNS. The analysis of the experimental and numerical results shows that under the investigated conditions the statistical trends describing the flow and flame structure are qualitatively similar. Specifically, a burner-attached flame is established in the stagnation region just upstream of the windward shear layer and in the low velocity region behind the jet 
potential core. While jet flames are nominally axisymmetric in the absence of crossflow, significant asymmetry is observed between the windward and leeward $O H$ layer thickness due to the substantially different scalar dissipation rates prevailing on the two sides of the jet. Vorticity spectra extracted from the windward shear layer reveal that the reacting jet is characterized by strong high-frequency, narrow-band oscillations at two frequencies, including a fundamental mode whose Strouhal number of $\sim 0.7$ agrees well with previous JICF stability studies in the same flow regime. The paper concludes with an analysis of the ignition, flame characteristics, and global structure of the burner-attached flame. Chemical explosive mode analysis (CEMA) shows that the the mixture on both sides of the jet will proceed to thermal runaway in isolation prior to ignition and the burning occurs in the diffusion mode after ignition. The predominantly mixing limited nature of the flow after ignition is supported by computing the Takeno flame index, which shows that $\sim 70 \%$ of the heat release occurs in non-premixed regions.

\section{Acknowledgements}

The work at Sandia National Laboratories was sponsored by the US Department of Energy, Office of Basic Energy Sciences, Division of Chemical Sciences, Geosciences, and Biosciences. The research used computing resources of the OLCF at Oak Ridge National Laboratory under the DOE INCITE program and the National Energy Research Scientific Computing Center. The work at Georgia Tech was partially supported by the Air Force Office of Scientific Research (contract \#FA9550-12-1-0107/RC657, contract monitor Dr. Chiping Lee) and the National Science Foundation (contract CBET-1235779, contract monitor, Professor Ruey-Hung Chen). The authors would also like to thank Prof. Ann Karagozian for generously sharing the contoured nozzle profile used in this work. The authors are grateful for the assistance of Ianko Chterev during setup of the diagnostic hardware. 


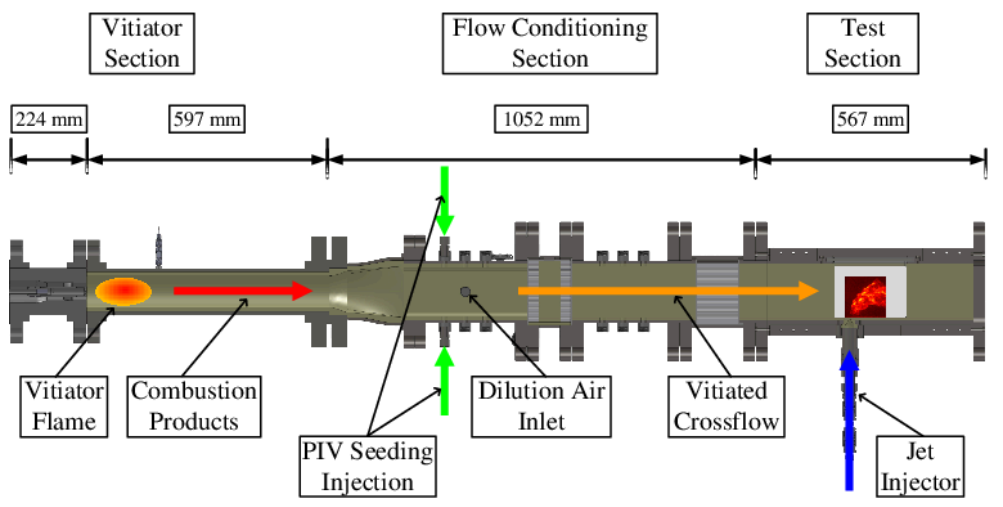

Figure 1: Schematic of the Reacting JICF Facility 


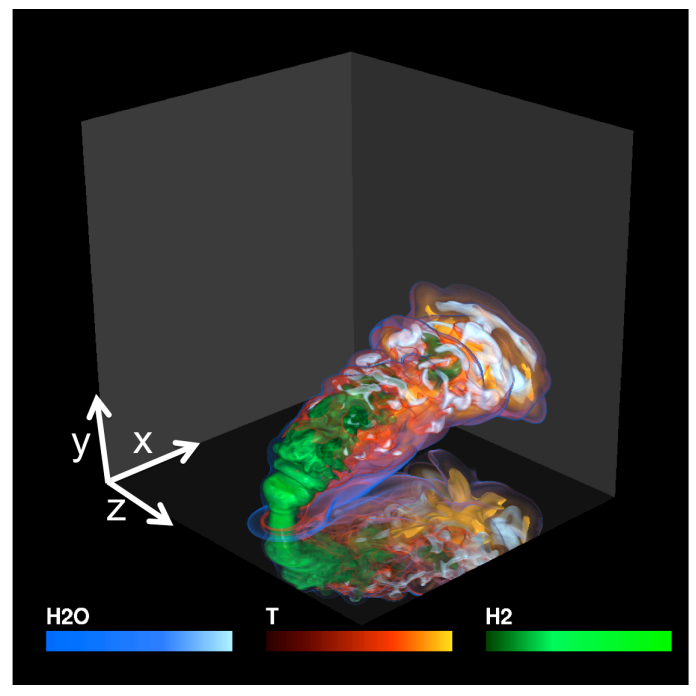

Figure 2: Volume rendering of the instantaneous temperature, $\mathrm{H}_{2} \mathrm{O}$ and $\mathrm{H}_{2}$ mass fraction fields.
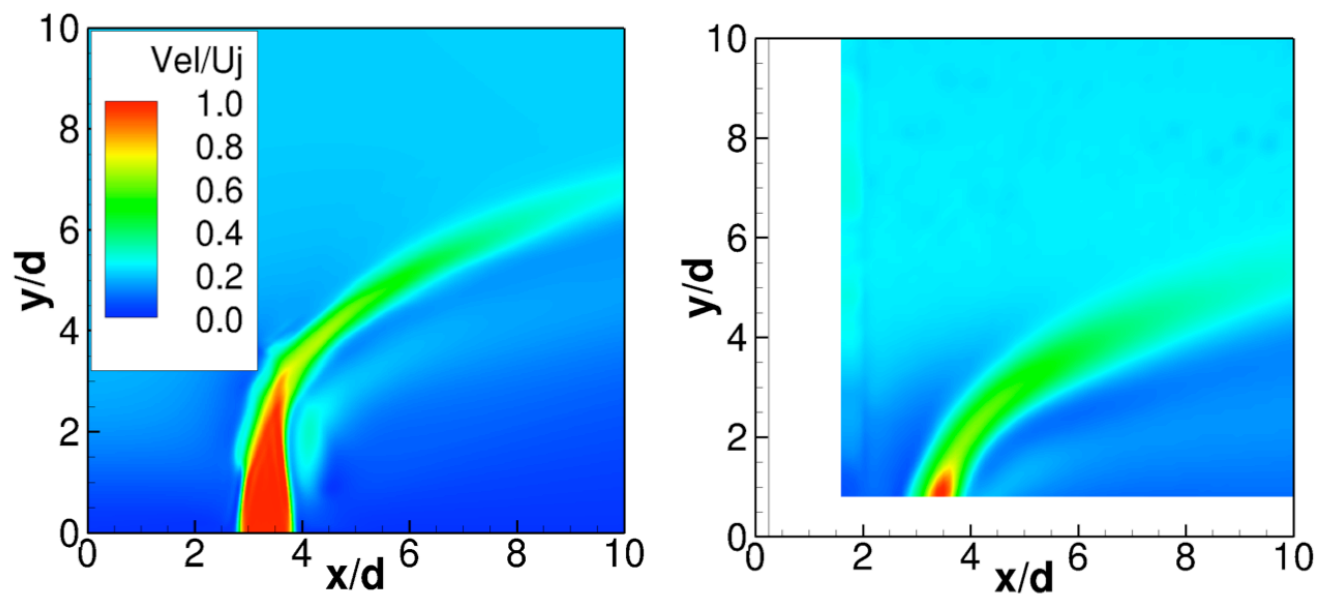

Figure 3: Reynolds-averaged velocity magnitude normalized by $U_{j}$ from the inert JICF, left: DNS, right: experiment. 

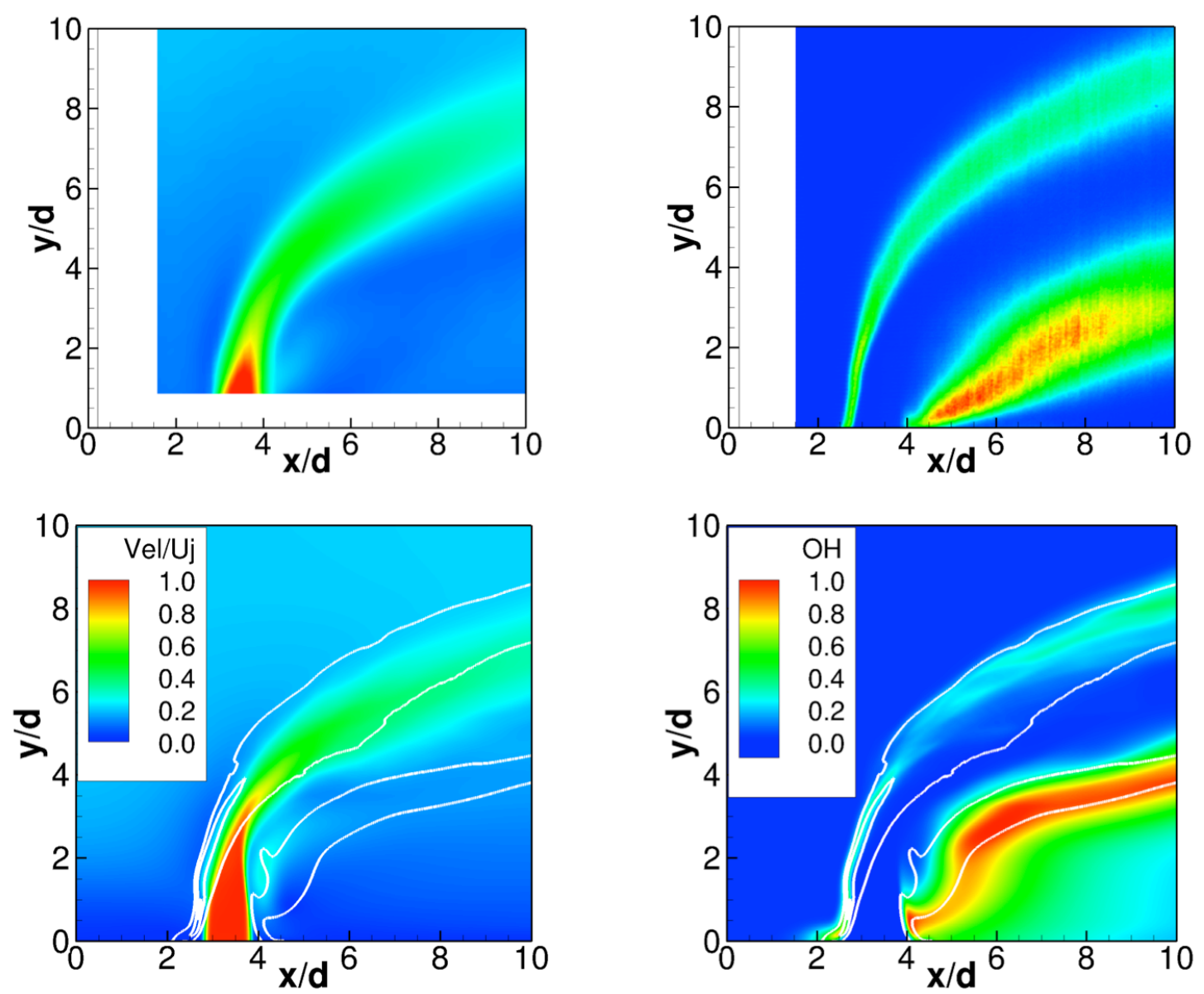

Figure 4: Reynolds-averaged velocity magnitude normalized by $U_{j}$ (left) and normalized $\mathrm{OH}$ mass fraction (right), top: experiment, bottom: DNS from the reacting JICF. Isolines of the mean heat release rate between $10 \%-90 \%$ of the maximum value (white) are overlaid on the DNS. The normalized OH-PLIF signal is plotted from the experiment. 

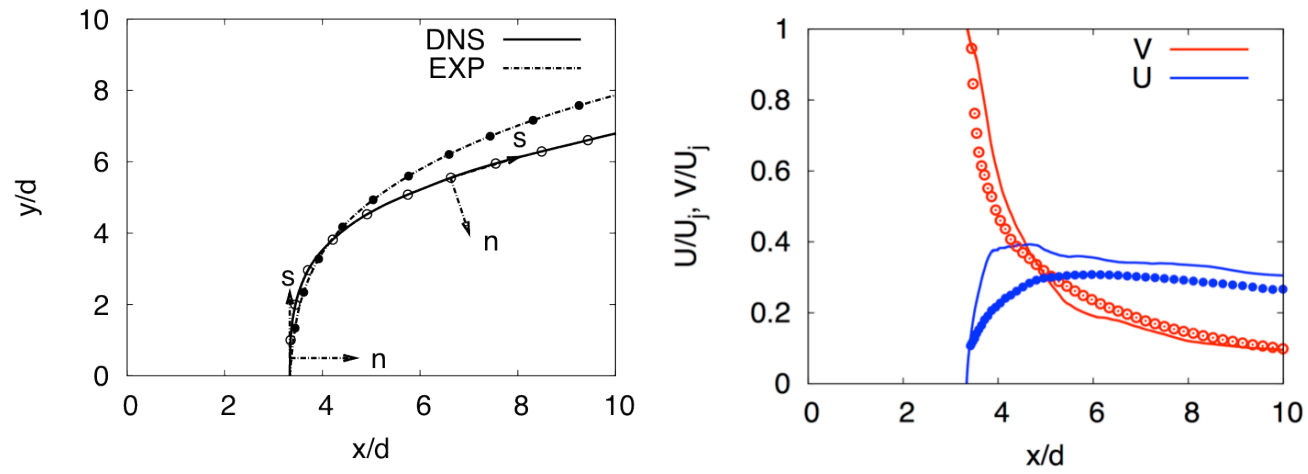

Figure 5: Left: Reynolds-averaged reacting jet trajectory. Solid line: DNS, Dashed line: experiment. Right: Reynolds-averaged streamwise and transverse velocity components normalized by $U_{j}$ on the jet trajectory. Lines: DNS, points: experiment.

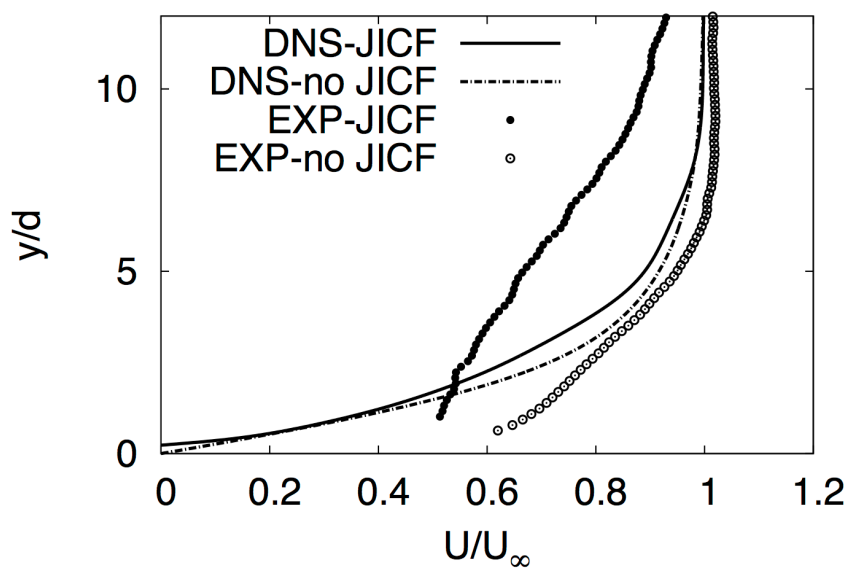

Figure 6: Normalized streamwise velocity profile on the spanwise midplane from the experiment (points) and the DNS (lines). Results are shown from reacting experiments and DNS with (solid line, closed symbols) and without (dotted dashed line, open symbols) the transverse jet. 

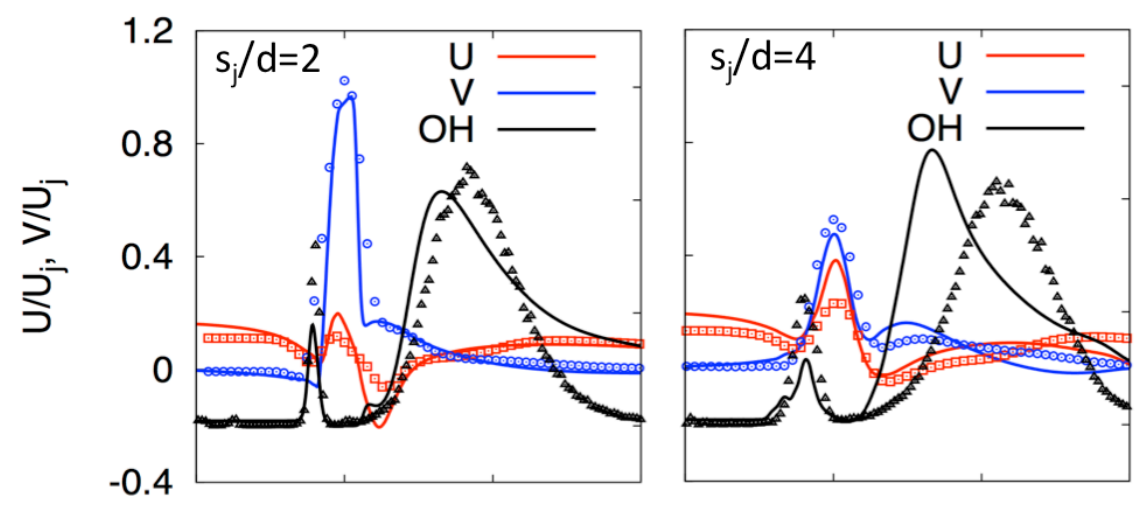

1.2
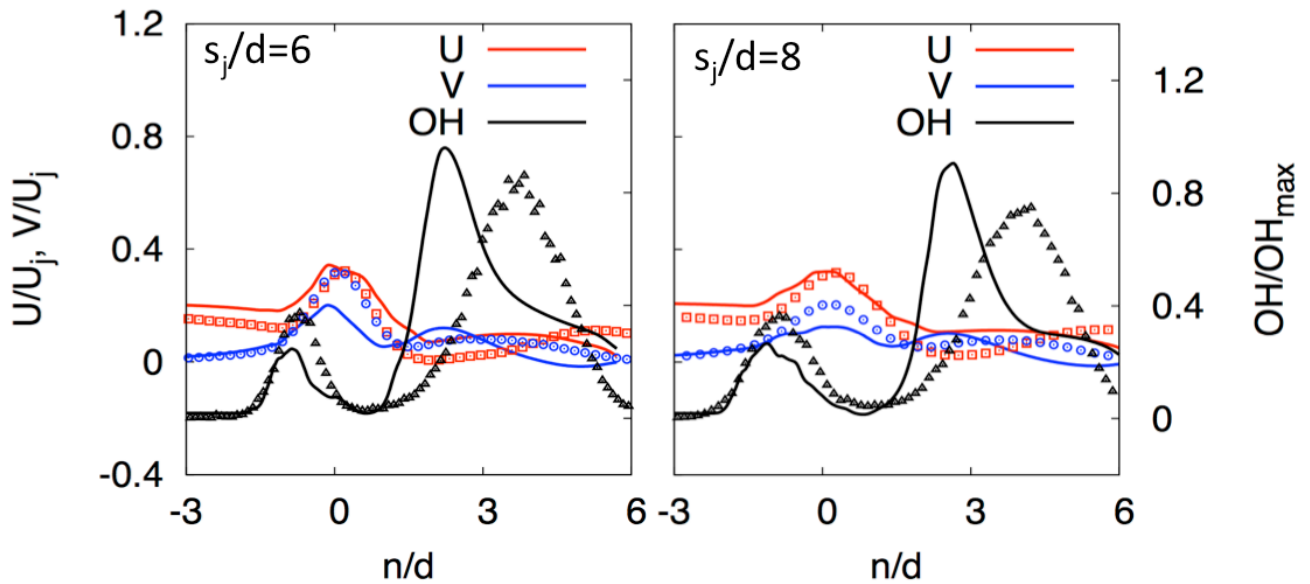

Figure 7: Reynolds-averaged streamwise velocity, transverse velocity and $O H$ mass fraction. Profiles are plotted as a function of the normal to the reacting jet $n$ coordinate at selected locations along the jet trajectory, $s_{j}$, as indicated on each figure. Lines: DNS, points: experiment. The normalized OH-PLIF signal is plotted from the experiment. 

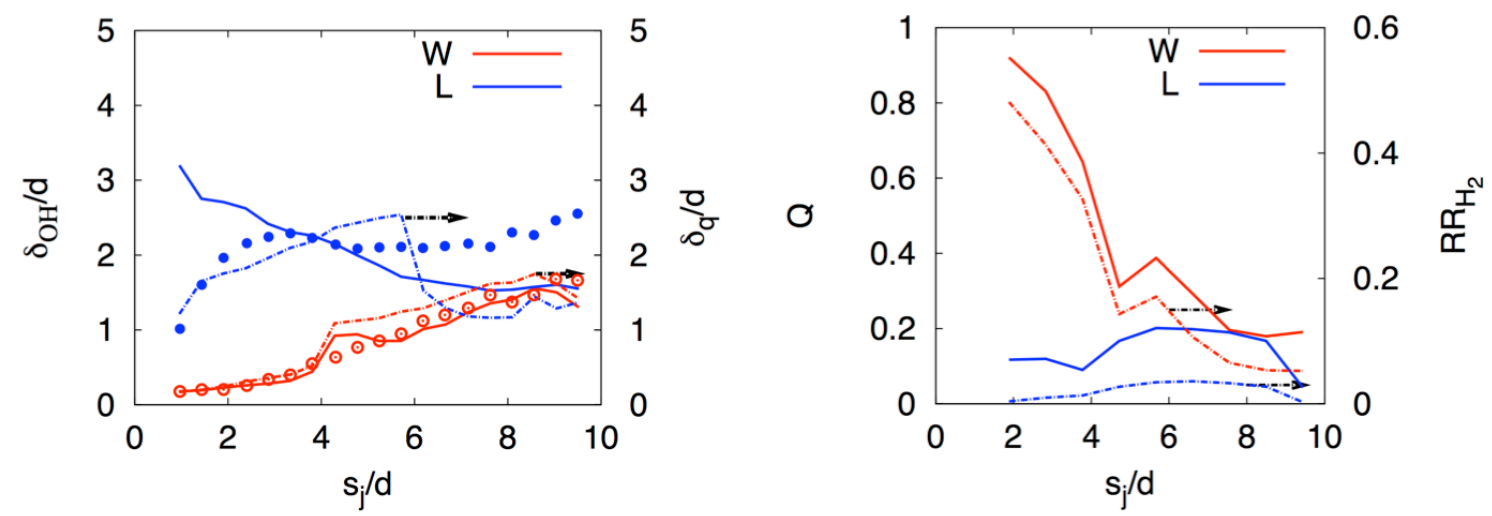

Figure 8: Left: Normalized $O H$ layer thickness $\left(\delta_{O H} / d\right)$ and heat release layer thickness $\left(\delta_{q} / d\right)$ from the DNS (lines) and normalized OH-PLIF $\left(\delta_{O H} / d\right)$ layer thickness from the experiment (points) as a function of the jet trajectory $s_{j}$ evaluated on the windward and leeward sides of the reacting jet. Right: Line integrated heat release rate and fuel consumption rate (right) as a function of the jet trajectory $s_{j}$ evaluated on the windward and leeward sides of the reacting jet. 

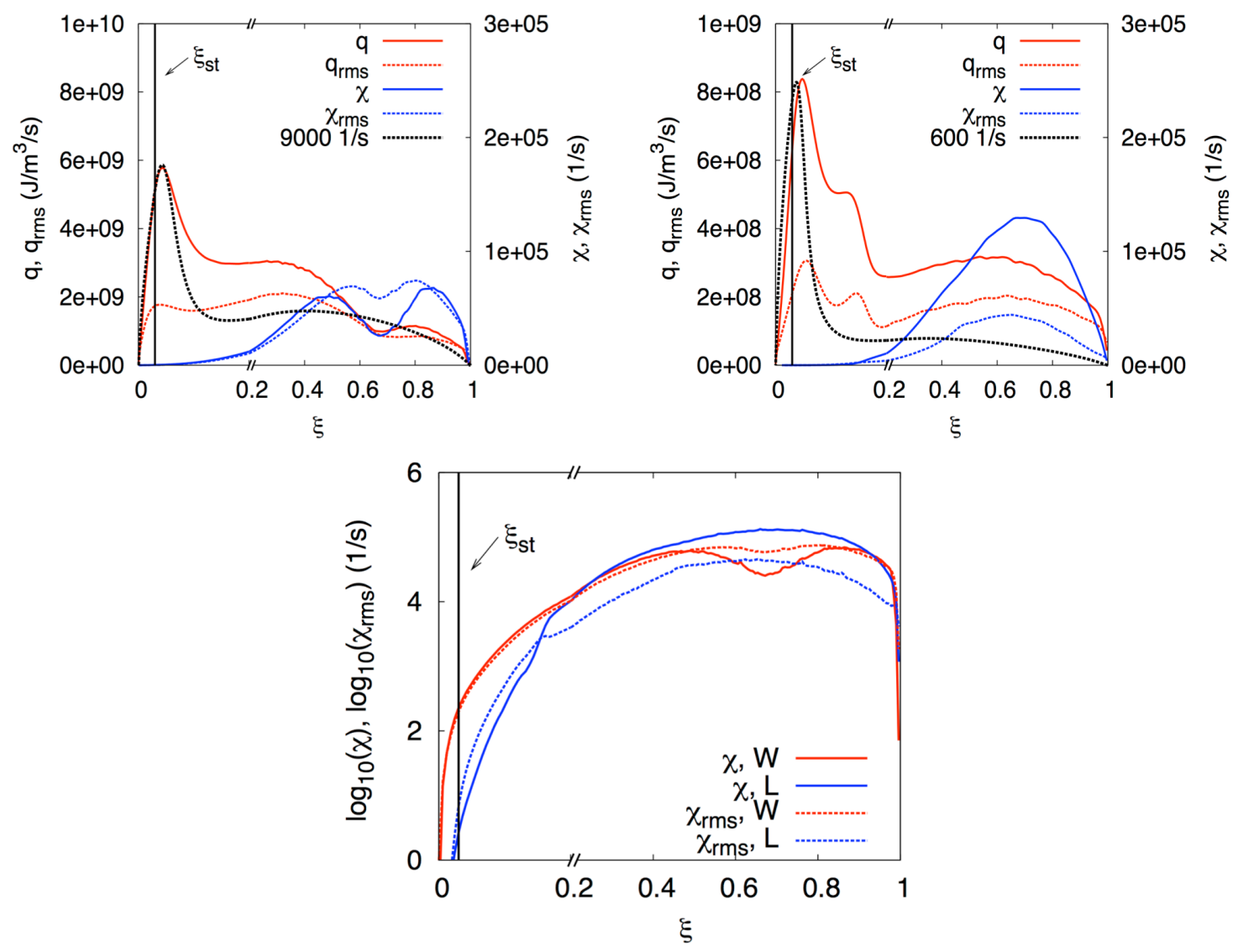

Figure 9: Top: Conditional means (solid lines) and rms (dashed lines) of the heat release rate (red) and scalar dissipation rate (blue) on the windward (left) and leeward (right) sides of the reacting jet. The heat release rate profiles of two strained flames at $5001 / \mathrm{s}$ and $90001 / s$, respectively are shown in black. Bottom: Conditional mean (solid lines) and rms (dashed lines) of the mixture fraction scalar dissipation rate on the windward (red) and leeward (blue) sides of the reacting jet. 

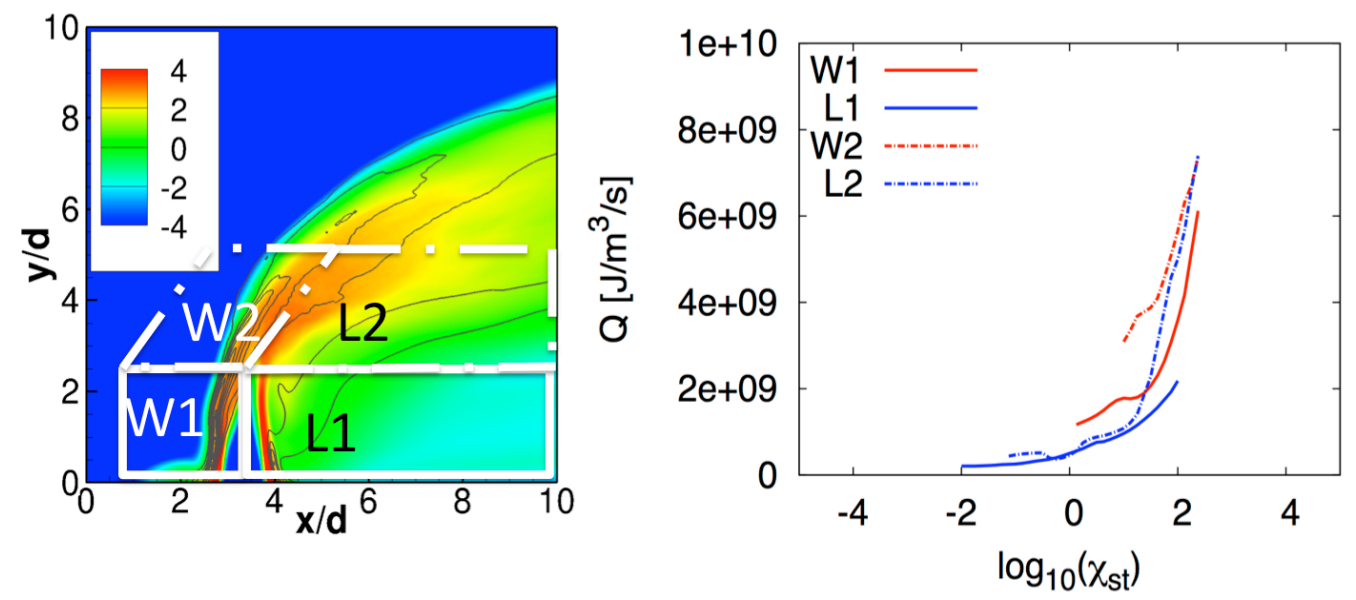

Figure 10: Reynolds-average of the logarithm of the mixture fraction dissipation rate (left). Conditional average of the heat release rate on the stoichiometric mixture fraction dissipation rate. Conditional averages are sampled on the windward (W) and leeward (L) sides of the reacting jet, at $y / d<3$ (W1, L1) and $3<y / d<6$ (W2, L2) as denoted in the figure on the left. 

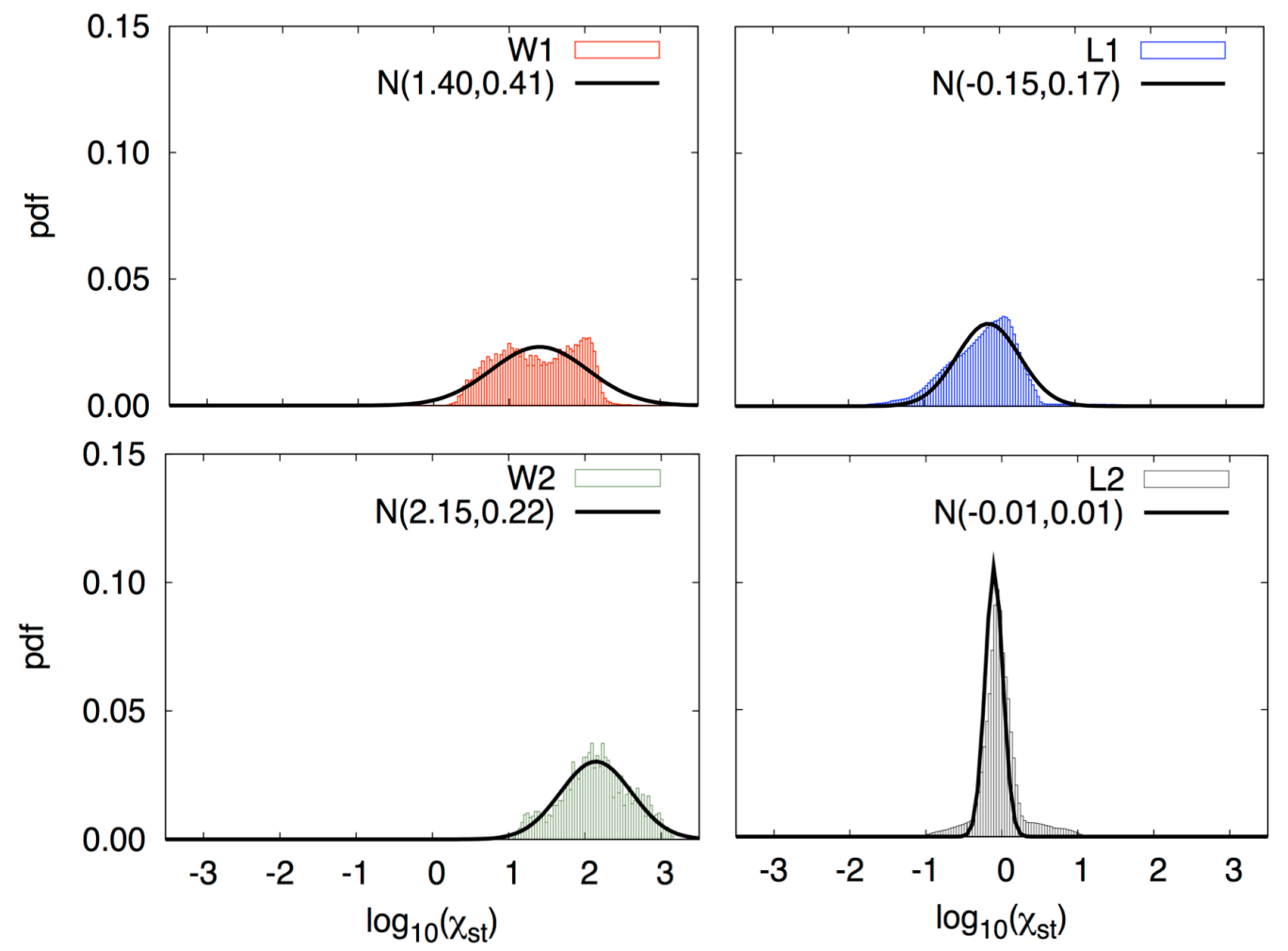

Figure 11: Pdf of the logarithm of the stoichiometric mixture fraction dissipation rate. Sampling is performed on the windward (W) and leeward (L) sides of the reacting jet, for $y / d<3(\mathrm{~W} 1, \mathrm{~L} 1)$ and $3<y / d<6$ (W2, L2). The solid lines represent normal distributions evaluated using the first two moments from the DNS data, denoted by the values in parentheses. 

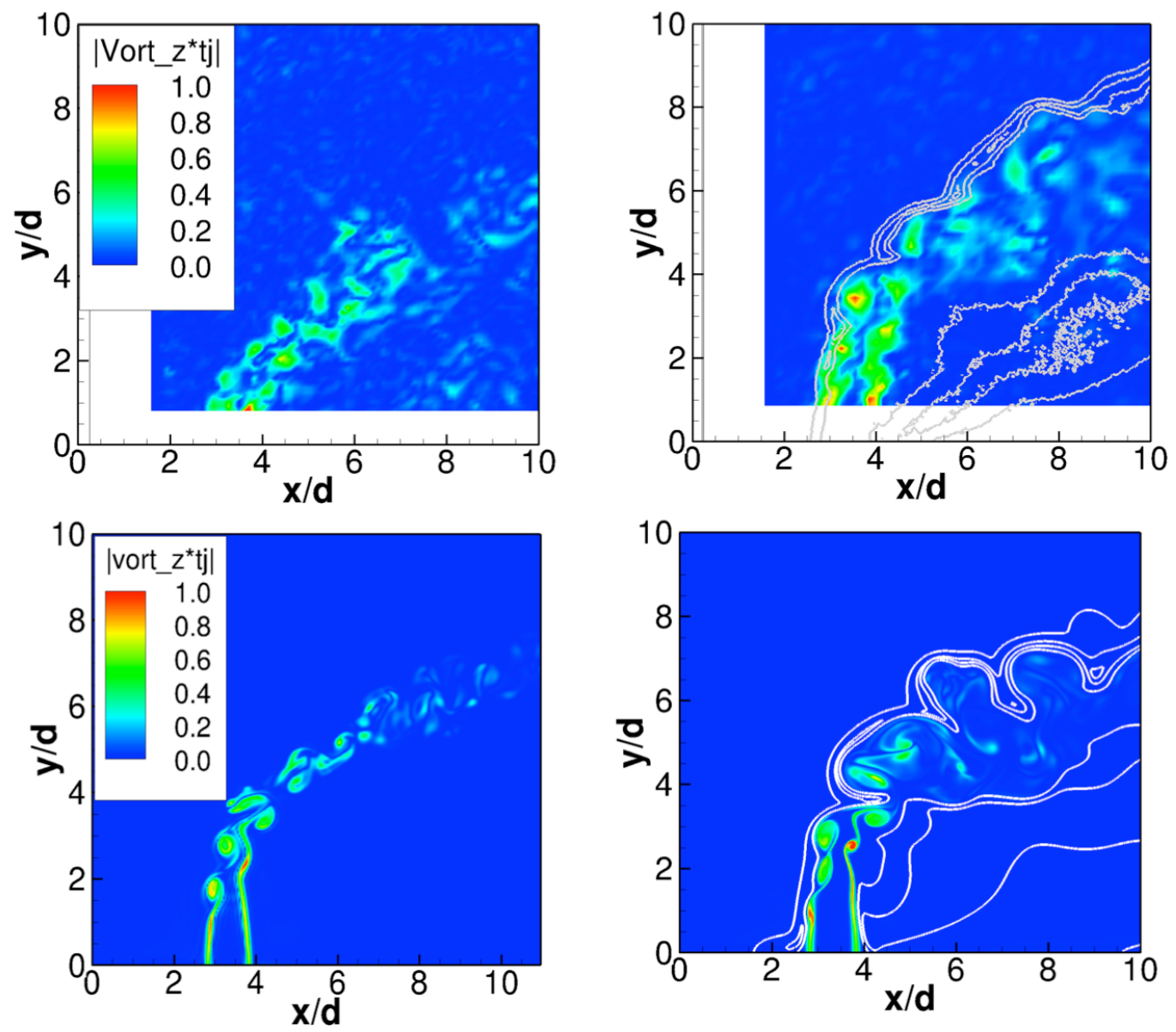

Figure 12: Spanwise vorticity component normalized by $t_{j}=d / U_{j}$ from the inert (left) and reacting (right) JICF, top: experiment, bottom: DNS. Isolines of the $O H$ mass fraction between $10 \%-90 \%$ of the maximum (white) demarcate the flame location. The normalized OH-PLIF signal is plotted from the experiment. 

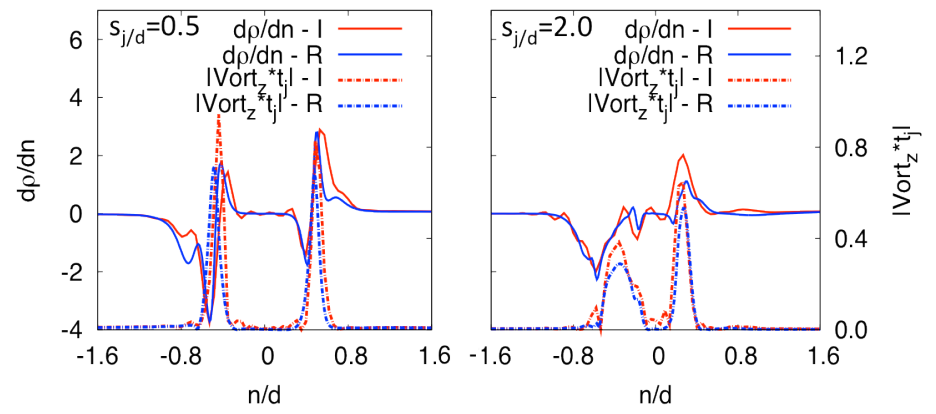

Figure 13: Reynolds-averaged density gradient and spanwise vorticity from the inert (I) and reacting (R) DNS. Profiles are plotted as a function of the normal to the jet $n$ coordinate at selected locations along the jet trajectory, $s_{j}$, as indicated on each figure. 

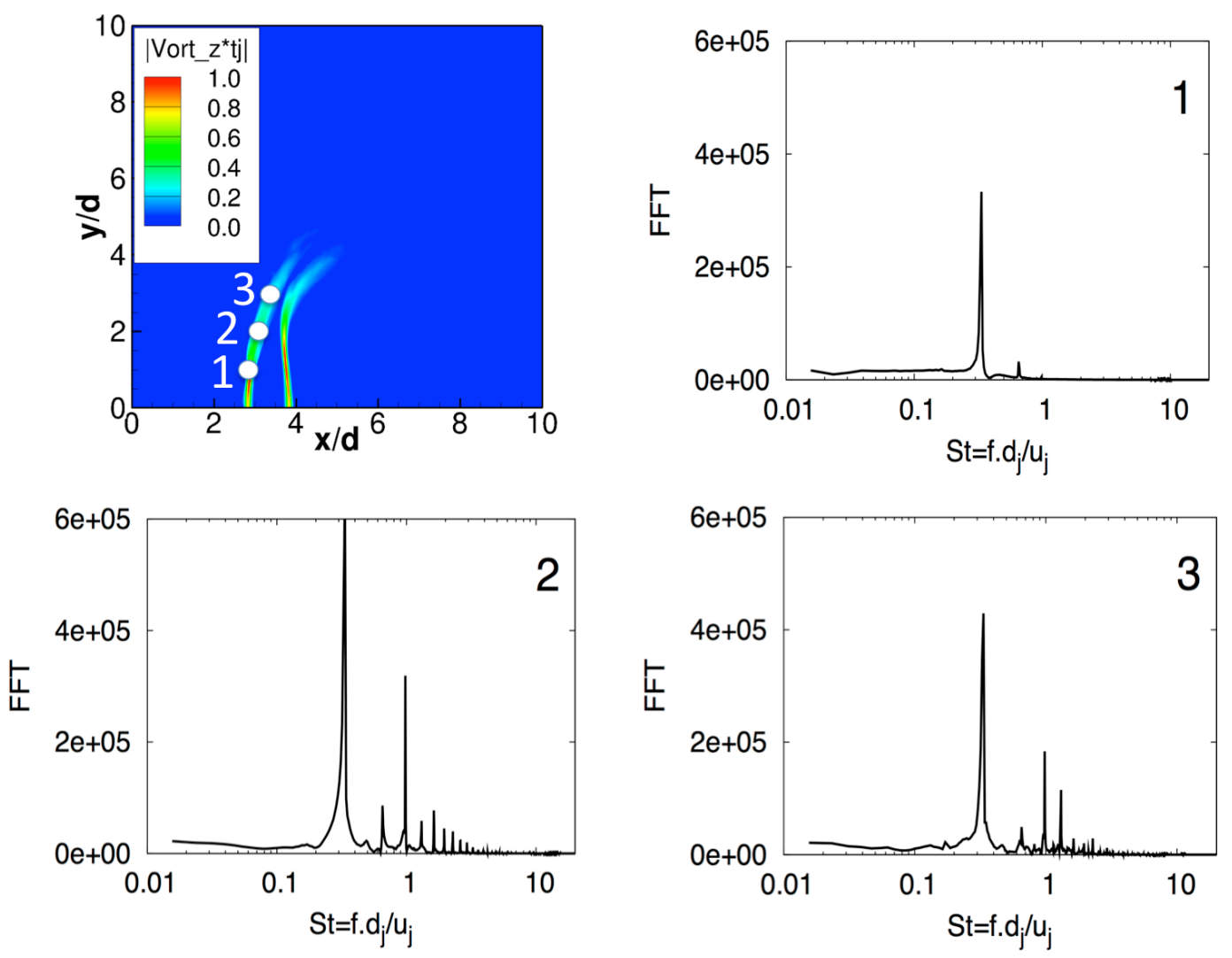

Figure 14: Probe locations and frequency spectra from probes 1, 2 and 3, located on the windward shear layer of the inert jet at $y / d=1,2$ and 3 , respectively. 

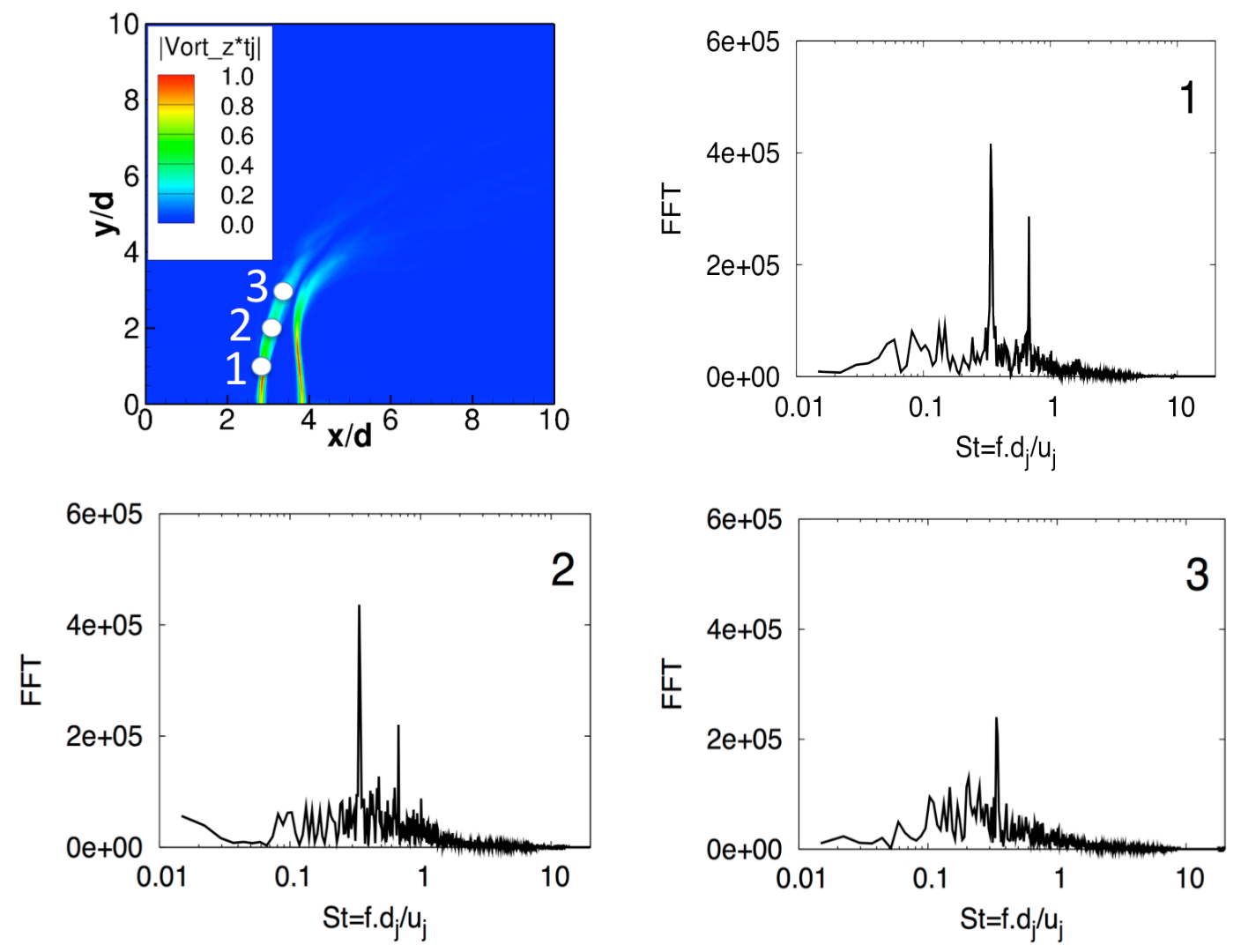

Figure 15: Probe locations and frequency spectra from probes 1, 2 and 3, located on the windward shear layer of the reacting jet at $y / d=1,2$ and 3 , respectively. 

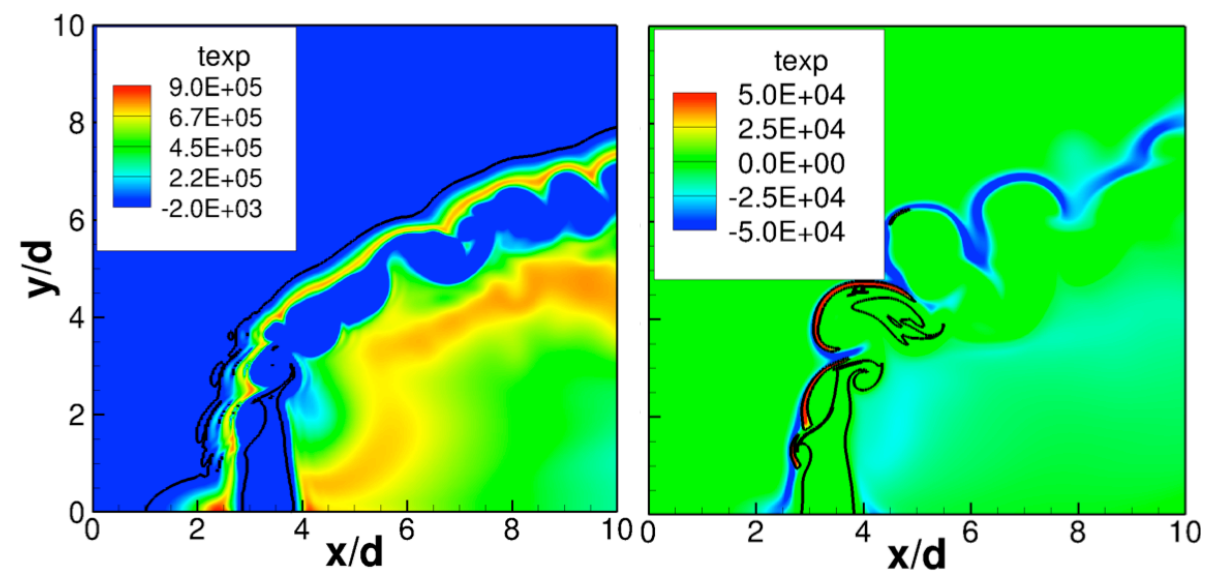

Figure 16: Chemical explosive mode $\left(t_{\text {exp }}\right)$ at the spanwise midplane from the inert (left) and reactive (right) DNS. The boundary between non-explosive and explosive regions is delineated by a black iso-contour at $t_{\exp }=0$.
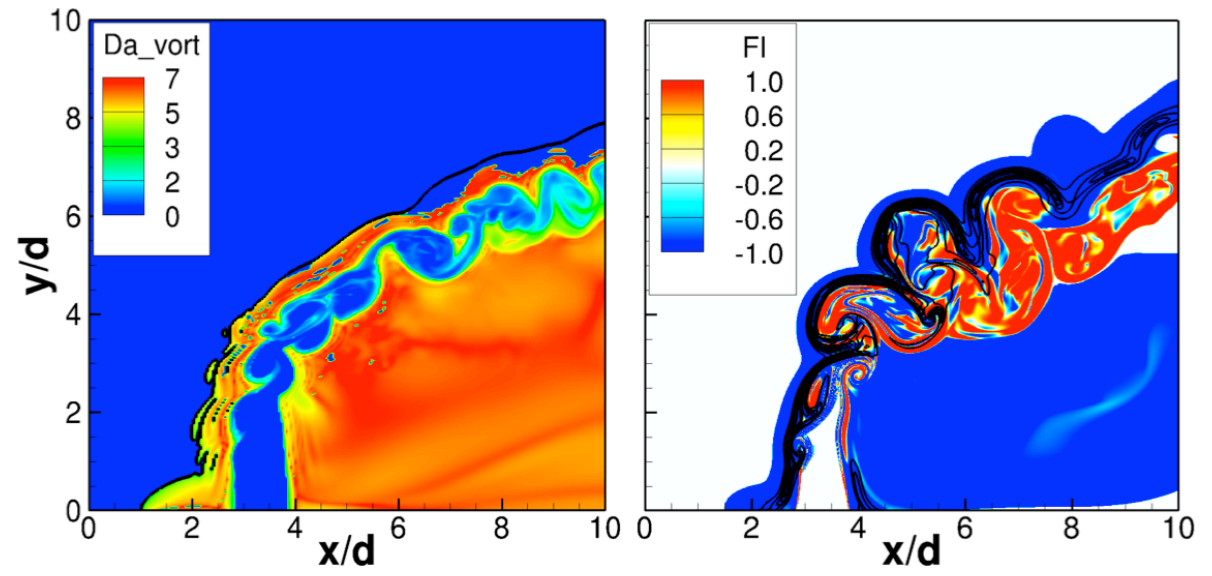

Figure 17: Left: Damköhler number at the spanwise midplane from the inert DNS. Right: Takeno flame index from the reacting DNS. isolines of the heat release rate, between $20 \%-80 \%$ of the peak (black) demarcate the reaction zone. 


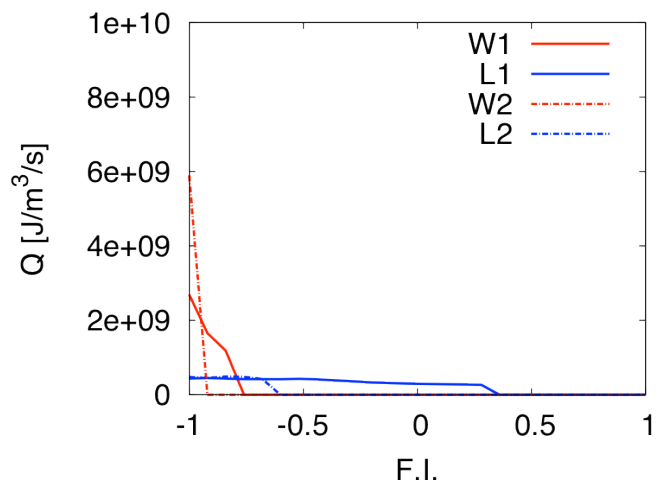

(a)
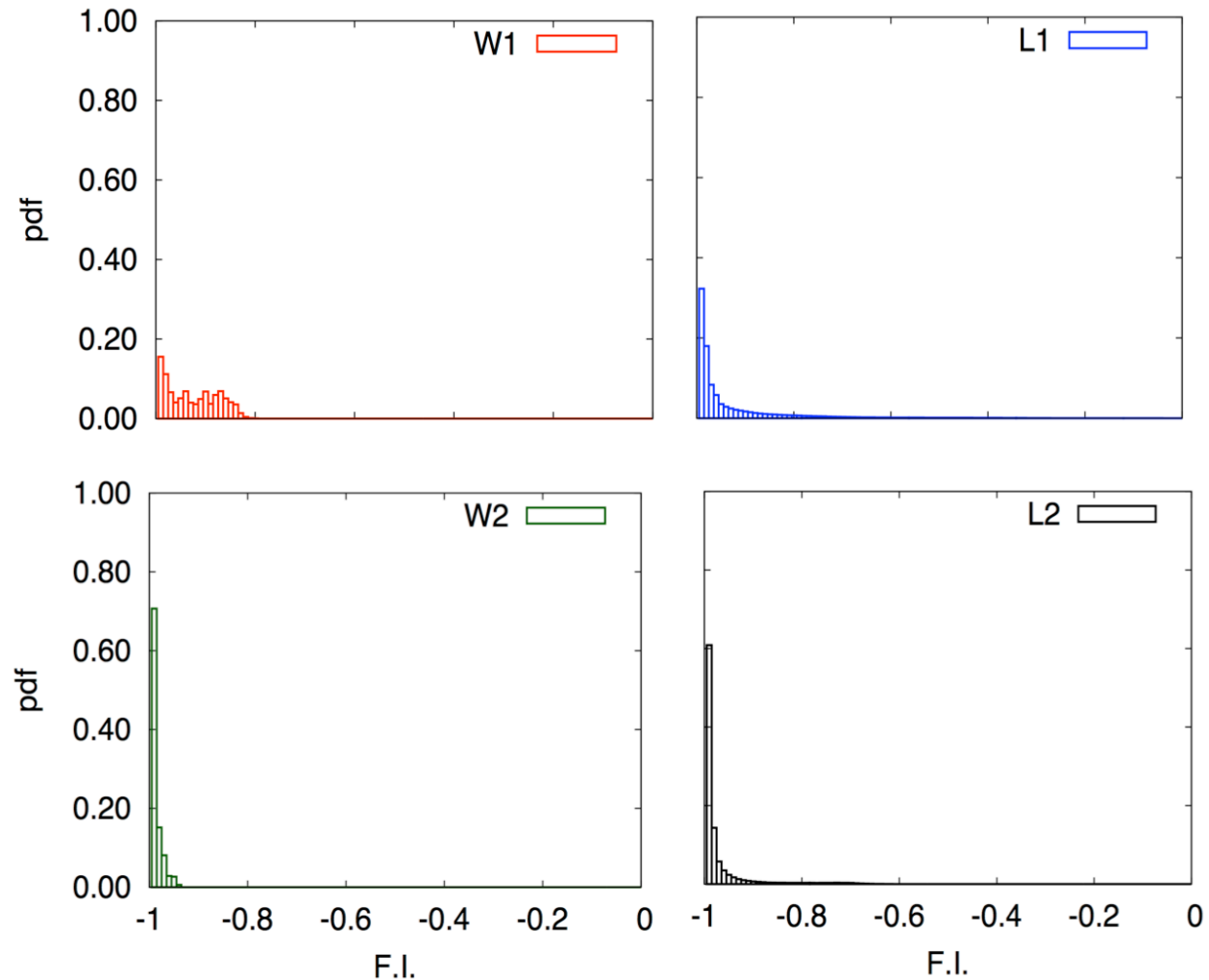

(b)

Figure 18: (a) Conditional average of the heat release rate on the Takeno flame index. (b) Pdf of the Takeno flame index. Averages are sampled on the windward (W) and leeward (L) sides of the reacting jet, for $y / d<3(\mathrm{~W} 1, \mathrm{~L} 1)$ and $3<y / d<6$ (W2, L2). 


\section{References}

[1] T. F. Fric, A. Roshko, J. Fluid Mech. 279 (1994) 1- 47.

[2] T. Lieuwen, Unsteady Combustor Physics, Cambridge University Press.

[3] A. R. Karagozian, Prog. in Energy and Comb. Sci. 36 (2010) 531-553.

[4] K. Mahesh, Ann. Rev. of Fluid Mech. 45 (1) (2013) 379-407.

[5] E. Hasselbrink, M. Mungal, J. Fluid Mech. 443 (2001) 27-68.

[6] J. K. Su, G. Mungal, J. Fluid Mech. 513 (2004) $1-45$.

[7] S. Muppidi, K. Mahesh, J. Fluid Mech. 530 (2005) 81-100.

[8] J. W. Shan, P. E. Dimotakis, J. Fluid Mech. 566 (2006) 47 - 96.

[9] S. Muppidi, K. Mahesh, J. Fluid Mech. 598 (2008) 335-360.

[10] M. Salewski, D. Stankovic, L. Fuchs, Turbulence Combust. 80 (2008) 255-283.

[11] A. M. Steinberg, R. Sadanandan, C. Dem, P. Kutne, W. Meier, Proc. Comb. Inst. 34 (2013) $1499-1507$.

[12] R. Grout, A. Gruber, H. Kolla, P. Bremer, J. Bennett, A. Gyulassy, J.H.Chen, J. Fluid Mech. 706 (2012) 351-383.

[13] H. Kolla, R. Grout, A. Gruber, J.H.Chen, Combust. Flame 159 (2012) 2255-2766.

[14] G. Kalghatgi, Comb. Sci. Tech. 26 (1981) $241-244$.

[15] G. Kalghatgi, Combust. Flame 52 (1983) $91-106$.

[16] R. Huang, J. Chan, Combust. Flame 98 (1994) 267 - 278.

[17] L. Kostiuk, A. Mejeski, P. Poudenx, M. Johnson, D. Wilson, Proc. Combust. Inst. 28 (1) (2000) 553-559.

[18] D. Micka, J. Driscoll, Combust. Flame 159 (2012) 1205-1214.

[19] J. Fleck, P. Griebel, A. Steinberg, C. Arndt, M. Aigner, Int. J. Hydrogen Energy 38 (2013) 16441-16452.

[20] R. Sullivan, B. Wilde, D. Noble, J. Seitzman, T. Lieuwen, Combust. Flame 161 (7) (2014) 1792-1803.

[21] S. Megerian, J. Davitian, L. de B. Alves, A. Karagozian, J. Fluid Mech. 593 (2007) $93-129$.

[22] S. Bagheri, P. Schlatter, P. Schmid, D. Henningson, S. Dan, J. Fluid Mech. 624 (2009) $33-44$. 
[23] P. Schlatter, S. Bagheri, D. Henningson, Theor. Comp. Fluid Dyn 25 (2011) 129-146.

[24] D. Getsinger, C. Hendrickson, A. Karagozian, Exp. Fluids 53 (2012) 783-801.

[25] P. A. Monkewitz, K. Sohn, AIAA journal 26 (8) (1988) 911 - 916.

[26] W. L. Chan, H. Kolla, J. Chen, M. Ihme, Combust. Flame 161 (2014) 2601-2613.

[27] J. Chen, A. Choudhary, B. de Supinski et. al., Comput. Sci. Discov. 2 (1) (2009) $1-31$.

[28] R. Grout, A. Gruber, C. Yoo, J.H.Chen, Proc. Comb. Inst. 33 (2012) 1629-1637.

[29] H. Kolla, R. Grout, A.Gruber, J. Chen, 8th US National Combustion Meeting, Park City, UT.

[30] E. Hawkes, J. Chen, Proc. Combust. Inst. 30 (2005) $647-655$.

[31] R. Sankaran, E. Hawkes, J. Chen, T. Lu, C. Law, Proc. Combust. Inst. 31 (2007) $1291-1298$.

[32] A. Gruber, R. Sankaran, E. Hawkes, J. Chen, J. Fluid Mech. 658 (2010) 5 - 32.

[33] E. Hawkes, R. Sankaran, J. Sutherland, J. Chen, Proc. Combust. Inst. 31 (2007) 1633 -1640 .

[34] C. Yoo, R. Sankaran, J. Chen, e, J. Fluid Mech. 640 (2009) 453-481.

[35] T. Echekki, G. Chen, Combust. Flame 134 (2003) 169 - 191.

[36] R. Sankaran, H. Im, E. Hawkes, J. Chen, Proc. Combust. Inst. 30 (2005) 875 - 882.

[37] R. J. Kee, F. Rupley, E. Meeks, J. Miller, Report No. SAND96-8216, Sandia National Laboratories, 1996.

[38] R. J. Kee, G. Dixon-Lewis, J. Warnatz, M. Coltrin, J. Miller, Report No. SAND868246, Sandia National Laboratories, 1986.

[39] J. Li, Z. Zhao, A. Kazakov, F. Dryer, Int. J. Chem. Kinet. 36 (2004) $566-575$.

[40] R. Moser, J. Kim, N. Mansour, Phys. Fluids (1994-present) 11 (4) (1999) 943-945.

[41] A. Lutz, R. Kee, J. Grcar, F. Ruply, Tech. Rep., SAND96-8243, Sandia National Laboratories.

[42] N. Peters, Comb. Sci. Tech. 30 ((1-6)) (1983) 1-17.

[43] N. Clemens, P. Paul, Combust. Flame 102 (3) (1995) $271-284$.

[44] R. Erickson, M. Soteriou, Combust. Flame 158 (12) (2011) 2441-2457.

[45] B. Emerson, J. O'Connor, M. Juniper, T. Lieuwen, J. Fluid Mech. (2012) 1-32.

[46] K. Tacina, W. J. A. Dahm, J. Fluid Mech. 415 (2000) 23 - 44. 
[47] P. E. Dimotakis, AIAA journal 24 (11) (1986) 1791-1796.

[48] M. C. Soteriou, A. F. Ghoniem, Phys. Fluids (1994-present) 7 (8) (1995) 2036-2051.

[49] P. Huerre, P. A. Monkewitz, ARFM 22 (1990) 473-537.

[50] K. Sreenivasan, S. Raghu, D. Kyle, Exp. Fluids 7 (5) (1989) 309-317.

[51] M.-H. Yu, P. A. Monkewitz, Phys. Fluids A: Fluid Dynamics (1989-1993) 2 (7) (1990) $1175-1181$.

[52] L. Raynal, J.-L.Harion, M. Favre-Marinet, G. Binder, Phys. Fluids (1994-present) 8 (4) (1996) 993-1006.

[53] T. Lu, C. Yoo, J. Chen, C. Law, J. Fluid Mech. 652 (2010) 45-64.

[54] H. Yamashita, M. Shimada, T. Takeno, Proc. Comb. Inst. 26 (1996) 24 -34. 


\section{List of Figures}

1 Schematic of the Reacting JICF Facility . . . . . . . . . . . . . . . . . . . . 24

2 Volume rendering of the instantaneous temperature, $\dot{H}_{2} \mathrm{O}$ and $\dot{H}_{2}$ mass fraction fields. . . . . . . . . . . . . . . . . . 25

3 Reynolds-averaged velocity magnitude normalized by $\dot{U}_{j}$ from the inert JICF, left: DNS, right: experiment. . . . . . . . . . . . . 25

4 Reynolds-averaged velocity magnitude normalized by $U_{j}$ (left) and normalized $O H$ mass fraction (right), top: experiment, bottom: DNS from the reacting JICF. Isolines of the mean heat release rate between $10 \%-90 \%$ of the maximum value (white) are overlaid on the DNS. The normalized OH-PLIF signal is plotted from the experiment.

5 Left: Reynolds-averaged reacting jet trajectory. Solid line: DNS, Dashed line: experiment. Right: Reynolds-averaged streamwise and transverse velocity components normalized by $U_{j}$ on the jet trajectory. Lines: DNS, points: experiment. . . . . . . . . . . . . . . . .

6 Normalized streamwise velocity profile on the spanwise midplane from the experiment (points) and the DNS (lines). Results are shown from reacting experiments and DNS with (solid line, closed symbols) and without (dotted dashed line, open symbols) the transverse jet. . . . . . . . . . . . .

7 Reynolds-averaged streamwise velocity, transverse velocity and $O H$ mass fraction. Profiles are plotted as a function of the normal to the reacting jet $n$ coordinate at selected locations along the jet trajectory, $s_{j}$, as indicated on each figure. Lines: DNS, points: experiment. The normalized OH-PLIF signal is plotted from the experiment. . . . . . . . . . . . . . .

8 Left: Normalized $O H$ layer thickness $\left(\delta_{O H} / d\right)$ and heat release layer thickness $\left(\delta_{q} / d\right)$ from the DNS (lines) and normalized OH-PLIF $\left(\delta_{O H} / d\right)$ layer thickness from the experiment (points) as a function of the jet trajectory $s_{j}$ evaluated on the windward and leeward sides of the reacting jet. Right: Line integrated heat release rate and fuel consumption rate (right) as a function of the jet trajectory $s_{j}$ evaluated on the windward and leeward sides of the reacting jet. . . . . . . . . . . . . . . .

9 Top: Conditional means (solid lines) and rms (dashed lines) of the heat release rate (red) and scalar dissipation rate (blue) on the windward (left) and leeward (right) sides of the reacting jet. The heat release rate profiles of two strained flames at $5001 / s$ and $90001 / s$, respectively are shown in black. Bottom: Conditional mean (solid lines) and rms (dashed lines) of the mixture fraction scalar dissipation rate on the windward (red) and leeward (blue) sides of the reacting jet. . . . . . . . . . . . . . . . .

10 Reynolds-average of the logarithm of the mixture fraction dissipation rate (left). Conditional average of the heat release rate on the stoichiometric mixture fraction dissipation rate. Conditional averages are sampled on the windward $(\mathrm{W})$ and leeward $(\mathrm{L})$ sides of the reacting jet, at $y / d<3$ (W1, L1) and $3<y / d<6(\mathrm{~W} 2, \mathrm{~L} 2)$ as denoted in the figure on the left. . . . . . 31

11 Pdf of the logarithm of the stoichiometric mixture fraction dissipation rate. Sampling is performed on the windward (W) and leeward (L) sides of the reacting jet, for $y / d<3(\mathrm{~W} 1, \mathrm{~L} 1)$ and $3<y / d<6(\mathrm{~W} 2, \mathrm{~L} 2)$. The solid lines represent normal distributions evaluated using the first two moments from the DNS data, denoted by the values in parentheses. . . . . . . . 
12 Spanwise vorticity component normalized by $t_{j}=d / U_{j}$ from the inert (left) and reacting (right) JICF, top: experiment, bottom: DNS. Isolines of the $\mathrm{OH}$ mass fraction between $10 \%-90 \%$ of the maximum (white) demarcate the flame location. The normalized OH-PLIF signal is plotted from the experiment. . . . . . . . . . . . . . . .

13 Reynolds-averaged density gradient and spanwise vorticity from the inert (I) and reacting (R) DNS. Profiles are plotted as a function of the normal to the jet $n$ coordinate at selected locations along the jet trajectory, $s_{j}$, as

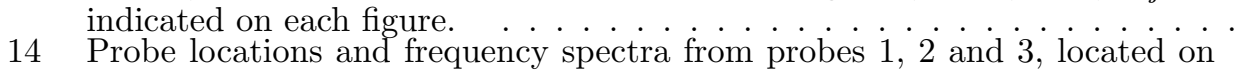
the windward shear layer of the inert jet at $y / d=1,2$ and 3 , respectively.

15 Probe locations and frequency spectra from probes 1, 2 and 3, located on the windward shear layer of the reacting jet at $y / d=1,2$ and 3 , respectively. 36

16 Chemical explosive mode $\left(t_{\text {exp }}\right)$ at the spanwise midplane from the inert (left) and reactive (right) DNS. The boundary between non-explosive and explosive regions is delineated by a black iso-contour at $t_{\exp }=0 . \ldots 37$

17 Left: Damköhler number at the spanwise midplane from the inert DNS. Right: Takeno flame index from the reacting DNS. isolines of the heat release rate, between $20 \%-80 \%$ of the peak (black) demarcate the reaction

18 (a) Conditional average of the heat release rate on the Takeno flame index. (b) Pdf of the Takeno flame index. Averages are sampled on the windward (W) and leeward (L) sides of the reacting jet, for $y / d<3$ (W1, L1) and $3<y / d<6(\mathrm{~W} 2, \mathrm{~L} 2) . \ldots \ldots \ldots \ldots \ldots \ldots$ 